\title{
Chiral Properties of Baryon Fields with Flavor $S U(3)$ Symmetry
}

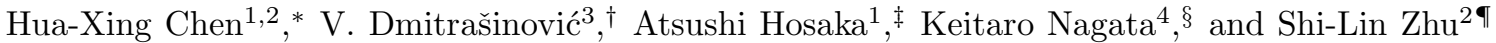 \\ ${ }^{1}$ Research Center for Nuclear Physics, Osaka University, Ibaraki 567-0047, Japan \\ ${ }^{2}$ Department of Physics, Peking University, Beijing 100871, China \\ 3 Vinča Institute of Nuclear Sciences, lab 010, P.O.Box 522, 11001 Beograd, Serbia \\ 4 Department of Physics, Chung-Yuan Christian University, Chung-Li 320, Taiwan
}

\begin{abstract}
We investigate chiral properties of local (non-derivative) fields of baryons consisting of three quarks with flavor $S U(3)$ symmetry. We construct explicitly independent local three-quark fields belonging to definite Lorentz and flavor representations. Chiral symmetry is spontaneously broken and therefore the baryon fields can have different chiral representations. It turns out that the allowed chiral representations are strongly correlated with the Lorentz group representations due to the color and spatial structure of the local three-quark fields. We discuss some implications of the allowed chiral symmetry representations on phenomenological Lagrangians with chiral $U(3)_{\mathrm{L}} \otimes U(3)_{\mathrm{R}}$ symmetry.
\end{abstract}

PACS numbers: 14.20.-c, 11.30.Rd, 11.40.Dw

Keywords: baryon, chiral symmetry, interpolating field

\section{INTRODUCTION}

As the chiral symmetry of QCD is spontaneously broken, $S U\left(N_{f}\right)_{L} \otimes S U\left(N_{f}\right)_{R} \rightarrow S U\left(N_{f}\right)_{V}\left(N_{f}\right.$ being the number of flavors), the observed hadrons are classified by the residual symmetry group representations of $S U\left(N_{f}\right)_{V}$. The full chiral symmetry may then conveniently be represented by its non-linear realization and this broken symmetry plays a dynamical role in the presence of the Nambu-Goldstone bosons and their interactions.

Yet, as pointed out by Weinberg [1], there are situations when it makes sense to consider algebraic aspects of chiral symmetry, i.e. the chiral multiplets of hadrons. Such hadrons may be classified in linear representations of the chiral symmetry group with some representations mixing. One such situation becomes realistic in the symmetry restored phase which is expected at high temperatures and/or densities [2]. If hadrons belong to certain representations of the chiral symmetry group, certain physical properties such as the axial coupling constants are determined by this symmetry. Therefore, the question as to what chiral representations, possibly with mixing, the hadrons belong to is of fundamental interest [3, 4, 5].

Another point of relevance is that the chiral representation can be used as a measure of the internal structure of hadrons. For instance, for a $\bar{q} q$ spin-one mesons, the possible chiral representations are $(\mathbf{8}, \mathbf{1})$ and $(\mathbf{3}, \overline{\mathbf{3}})$ and their left-right conjugates for flavor octet mesons. As a matter of fact, for the multiquark hadrons, the allowed chiral representations can be more complicated/higher dimensional with increasing number of quarks and antiquarks. Hence the study of chiral representations may provide some hints to the structure of hadrons, extending possibly beyond the minimal constituent picture [6, 7, [8, 9, 10, 11].

Motivated by this argument, we have recently performed a complete classification of baryon fields constructed from three quarks in the local form with two light flavors (the so-called $S U(2)$ sector) [12. Such baryon fields are used as interpolators for the study of two-point correlation functions in the QCD sum rule approach and in the lattice QCD [13, 14, 15, 16, 17, 18]. Strictly speaking, however, the chiral structure of an interpolator does not directly reflect that of the physical state when chiral symmetry is spontaneously broken. But the minimal configuration of three quarks provides at least a guide to the simplest expectations for baryons. Any deviation from such a simple structure may be an indication of higher Fock-space components, such as the multi-quark ones [19].

Another reason for such a study of chiral classifications is related to the number of independent fields. In principle, the correlation functions should contain all information about physical states when computed exactly. Practically, however, one must rely on some approximation, and it has been observed in previous studies, that the results may

\footnotetext{
*Electronic address: hxchen@rcnp.osaka-u.ac.jp

${ }^{\dagger}$ Electronic address: dmitrasin@yahoo.com

${ }^{\ddagger}$ Electronic address: hosaka@rcnp.osaka-u.ac.jp

$\S$ Electronic address: nagata@phys.cycu.edu.tw

IElectronic address: zhusl@phy.pku.edu.cn
} 
depend significantly on the choice of the interpolators, which are generally taken as linear combinations of the independent ones [5, 15, 20].

In this paper, we perform a complete classification of baryon fields written as local products (without derivatives) of three quarks according to chiral symmetry group $S U(3)_{L} \otimes S U(3)_{R}$. This is an extension of our previous work for the case of flavor $S U(2)$ [12]. Technically, the $S U(3)$ algebra introduces more complications, which makes insight less at work. Hence, here we attempt to explore a rather technical aspect which enables one to perform systematic classification. We derive general transformation rules for baryon fields for the classification, while maximally utilizing the Fierz transformations in order to implement the Pauli principle among the quarks. The objective of $S U(3)$ baryon fields provides a simple but suitable exercise how the method works. It can be extended to systems with more complex hadron fields containing more quarks [20].

As in the previous paper [12], we first establish the classification under the flavor $S U(3)$ symmetry, and then investigate the properties under chiral symmetry group. The method is based essentially on the tensor method for $S U(3)$, while the Fierz method for the Pauli principle associated with the structure in the color, flavor, Lorentz (spin) and orbital spaces is utilized when establishing the independent fields. It turns out that for local three-quark fields, the Pauli principle puts a constraint on the structure of the Lorentz and chiral representations. This leads essentially to the same permutation symmetry structure as in the case of flavor $S U(2)$.

This paper is organized as follows. In Section II we establish the independent local baryon interpolating fields, and investigate their flavor $S U(3)$ symmetry properties. In Section III, we investigate the properties of the baryon fields under chiral symmetry transformations $S U(3)_{L} \otimes S U(3)_{R}$. We find that both flavor and chiral symmetry properties are related to the structure of the Lorentz group. Eventually, in Section IV, we find that this can be explained by the Pauli principle for the left and right handed quarks, which puts a constraint on permutation symmetry properties of three-quarks. Some complicated formulae are shown in appendices.

\section{FLAVOR SYMMETRIES OF THREE-QUARK BARYON FIELDS}

Local fields for baryons consisting of three quarks can be generally written as

$$
B(x) \sim \epsilon_{a b c}\left(q_{A}^{a T}(x) C \Gamma_{1} q_{B}^{b}(x)\right) \Gamma_{2} q_{C}^{c}(x),
$$

where $a, b, c$ denote the colour and $A, B, C$ the flavour indices, $C=i \gamma_{2} \gamma_{0}$ is the charge-conjugation operator, $q_{A}(x)=$ $(u(x), d(x), s(x))$ is the flavour triplet quark field at location $x$, and the superscript $T$ represents the transpose of the Dirac indices only (the flavour and colour $S U(3)$ indices are not transposed). The antisymmetric tensor in color space $\epsilon_{a b c}$, ensures the baryons' being color singlets. The space-time coordinate $x$ does nothing with our studies, and we shall omit it. The matrices $\Gamma_{1,2}$ are Dirac matrices which describe the Lorentz structure. With a suitable choice of $\Gamma_{1,2}$ and taking a combination of indices of $A, B$ and $C$, the baryon operators are defined so that they form an irreducible representation of the Lorentz and flavour groups, as we shall show in this section.

We employ the tensor formalism for flavour $S U(3)$ a la Okubo [21, 22, 23, 24, 25] for the quark field $q$, although the explicit expressions in terms of up, down and strange quarks are usually employed in lattice QCD and QCD sum rule studies. We shall see that the tensor formulation simplifies the classification of baryons into flavour multiplets and leads to a straightforward (but generally complicated) derivation of Fierz identities and chiral transformations of baryon operators. This is in contrast with the $N_{f}=2$ case where we explicitly included isospin into the $\Gamma_{1,2}$ matrices and thus produced isospin invariant/covariant objects [12]. The reason for this switch is that in the $N_{f}=3$ case the baryons form octets and decuplets, rather than doublets and quartets, but the octet and decuplet projection operators cannot be easily introduced into this formalism.

\section{A. Flavour $S U(3)_{f}$ decomposition for baryons}

For the sake of notational completeness, we start with some definitions. The quarks of flavor $S U(3)$ form either the contra-variant $(\mathbf{3})$ or the covariant $(\overline{\mathbf{3}})$ fundamental representations. They are distinguished by either upper or lower index as

$$
\begin{aligned}
q^{A} \in q & =\left(\begin{array}{c}
u \\
d \\
s
\end{array}\right), \\
q_{A} \in q^{\dagger} & =\left(u^{*}, d^{*}, s^{*}\right) .
\end{aligned}
$$


The two conjugate fundamental representations transform under flavor $S U(3)$ transformations as

$$
\begin{aligned}
q & \rightarrow \exp \left(i \frac{\vec{\lambda}}{2} \vec{a}\right) q, \\
q^{\dagger} & \rightarrow q^{\dagger} \exp \left(-i \frac{\vec{\lambda}}{2} \vec{a}\right),
\end{aligned}
$$

where $a_{N}(N=1, \cdots, 8)$ are the octet of $S U(3)_{F}$ group parameters and $\lambda^{N}$ are the eight Gell-Mann matrices. Since the latter are Hermitian, we may replace the transposed matrices with the complex conjugate ones. The set of eight $\bar{\lambda}^{N}=-\left(\lambda^{N}\right)^{T}=-\left(\lambda^{N}\right)^{*}$ matrices form the generators of the irreducible $\overline{\mathbf{3}}$ representation.

Now for three quarks, we show flavor $S U(3)$ irreducible decomposition $\mathbf{3} \otimes \mathbf{3} \otimes \mathbf{3}=\mathbf{1} \oplus \mathbf{8} \oplus \mathbf{8} \oplus \mathbf{1 0}$ explicitly in terms of three quarks. It can be done by making suitable permutation symmetry representations of three-quark products $q_{A} q_{B} q_{C}$.

1. The totally antisymmetric combination which forms the singlet,

$$
\Psi_{[A B C]}=\mathcal{N}\left(q_{A} q_{B} q_{C}+q_{B} q_{C} q_{A}+q_{C} q_{A} q_{B}-q_{B} q_{A} q_{C}-q_{A} q_{C} q_{B}-q_{C} q_{B} q_{A}\right) .
$$

The normalization constant here is $\mathcal{N}=1 / \sqrt{6}$. In the quark model this corresponds to $\Lambda(1405)$. In order to represent this totally antisymmetric combination, we can use the totally antisymmetric tensor $\epsilon^{A B C}$. Then the flavor singlet baryon field $\Lambda$ can be written as:

$$
\Lambda \equiv \epsilon^{A B C} \epsilon_{a b c}\left(q_{A}^{a T} C \Gamma_{1} q_{B}^{b}\right) \Gamma_{2} q_{C}^{c}
$$

2. The totally symmetric combination which forms the decuplet,

$$
\Psi_{\{A B C\}}=\mathcal{N}\left(q_{A} q_{B} q_{C}+q_{B} q_{C} q_{A}+q_{C} q_{A} q_{B}+q_{B} q_{A} q_{C}+q_{A} q_{C} q_{B}+q_{C} q_{B} q_{A}\right)
$$

The normalization constant depends on the set of quarks for baryons: for $q_{A}, q_{B}, q_{C}=u, d, s, \mathcal{N}=1 / \sqrt{6}$, while it is $1 / 6$ for $q_{A}, q_{B}, q_{C}=u u u$. In order to represent this totally symmetric flavor structure, we introduce the totally symmetric tensor $S_{P}^{A B C}(P=1, \cdots, 10)$. Then the flavor decuplet baryon field $\Delta$ can be written as:

$$
\Delta^{P} \equiv S_{P}^{A B C} \epsilon_{a b c}\left(q_{A}^{a T} C \Gamma_{1} q_{B}^{b}\right) \Gamma_{2} q_{C}^{c}
$$

The non-zero components of $S_{P}^{A B C}(=1)$ are summarized in Table $\llbracket$ The rest of components are just zero, for instance, $S_{1}^{112}=0$.

TABLE I: Non-zero components of $S_{P}^{A B C}(=1)$

\begin{tabular}{c|cccccccccc}
\hline \hline$P$ & 1 & 2 & 3 & 4 & 5 & 6 & 7 & 8 & 9 & 10 \\
\hline$A B C$ & 111 & 112 & 113 & 122 & 123 & 133 & 222 & 223 & 233 & 333 \\
\hline Baryons & $\Delta^{++}$ & $\Delta^{+}$ & $\Sigma^{*+}$ & $\Delta^{0}$ & $\Sigma^{* 0}$ & $\Xi^{* 0}$ & $\Delta^{-}$ & $\Sigma^{*-}$ & $\Xi^{*-}$ & $\Omega^{-}$ \\
\hline
\end{tabular}

3. The two mixed symmetry tensors of the $\rho$ and $\lambda$ types are defined by

$$
\begin{aligned}
& \Psi_{[A\{B] C\}}^{\rho}=\mathcal{N}\left(2 q_{A} q_{B} q_{C}-q_{B} q_{C} q_{A}-q_{C} q_{A} q_{B}-2 q_{B} q_{A} q_{C}+q_{A} q_{C} q_{B}+q_{C} q_{B} q_{A}\right) \\
& \Psi_{\{A[B\} C]}^{\lambda}=\mathcal{N}\left(2 q_{A} q_{B} q_{C}-q_{B} q_{C} q_{A}-q_{C} q_{A} q_{B}+2 q_{B} q_{A} q_{C}-q_{A} q_{C} q_{B}-q_{C} q_{B} q_{A}\right) .
\end{aligned}
$$

Here the two symbols in \{\} are first symmetrized and then the symbols in [ ] are anti-symmetrized. The normalization constant depends again on the number of different kinds of terms. The correspondence of the octet fields of (8) and the physical ones can be made first by taking the following combinations

$$
\begin{aligned}
& N_{8 \rho}^{N}=\epsilon^{A B D}\left(\boldsymbol{\lambda}^{N}\right)_{D C} \Psi_{[A\{B] C\}}^{\rho}, \\
& N_{8 \lambda}^{N}=\epsilon^{B C D}\left(\boldsymbol{\lambda}^{N}\right)_{D A} \Psi_{\{A[B\} C]}^{\lambda} .
\end{aligned}
$$

This kind of "double index" ( $D C$ for $N_{8 \rho}^{N}$ and $D A$ for $N_{8 \lambda}^{N}$ ) notation for the baryon flavour has been used by Christos [26]. In our discussions, we shall use the following form for the flavor octet baryon field

$$
N^{N} \equiv \epsilon^{A B D}\left(\boldsymbol{\lambda}^{N}\right)_{D C} \epsilon_{a b c}\left(q_{A}^{a T} C \Gamma_{1} q_{B}^{b}\right) \Gamma_{2} q_{C}^{c}
$$


It is of the $\rho$ type. But after using Fierz transformations to interchange the second and the third quarks, the transformed one contains $\lambda$ type also, as we shall show in the following. The octet of physical baryon fields are then determined by

$$
\begin{aligned}
& N^{1} \pm i N^{2} \sim \Sigma^{\mp}, \quad N^{3} \sim \Sigma^{0}, \quad N^{8} \sim \Lambda, \\
& N^{4} \pm i N^{5} \sim \Xi^{-}, p, \quad N^{6} \pm i N^{7} \sim \Xi^{0}, n,
\end{aligned}
$$

or put into the $3 \times 3$ baryon matrix

$$
\mathfrak{B}=\left(\begin{array}{ccc}
\frac{\Sigma^{0}}{\sqrt{2}}+\frac{\Lambda^{8}}{\sqrt{6}} & \Sigma^{+} & p \\
\Sigma^{-} & -\frac{\Sigma^{0}}{\sqrt{2}}+\frac{\Lambda^{8}}{\sqrt{6}} & n \\
\Xi^{-} & \Xi^{0} & -\frac{2}{\sqrt{6}} \Lambda^{8}
\end{array}\right) .
$$

\section{B. Counting the (in)dependent fields}

In this section we investigate independent baryon fields for each Lorentz group representation which is formed by three quarks. The irreducible decomposition of the Lorentz group is done as

$$
\left(\left(\frac{1}{2}, 0\right) \oplus\left(0, \frac{1}{2}\right)\right)^{3} \sim\left(\left(\frac{1}{2}, 0\right) \oplus\left(0, \frac{1}{2}\right)\right) \oplus\left(\left(1, \frac{1}{2}\right) \oplus\left(\frac{1}{2}, 1\right)\right) \oplus\left(\left(\frac{3}{2}, 0\right) \oplus\left(0, \frac{3}{2}\right)\right)
$$

where we have ignored the multiplicity on the right hand side. The three representations are described by the Dirac spinor field, the Rarita-Schwinger's vector spinor field and the antisymmetric tensor spinor field, respectively. In order to establish independent fields when combined with color, flavor, and Lorentz (spin) degrees of freedom, we employ the method of Fierz transformations which are essentially equivalent to the use of the Pauli principle for three quarks. Here we demonstrate the essential idea for the simplest case for the Dirac spinor, $\left(\frac{1}{2}, 0\right) \oplus\left(0, \frac{1}{2}\right)$. Other cases are briefly explained in Appendices $\mathrm{A}$ and B.

\section{Flavor singlet baryon}

Let us start with writing down five baryon fields which contain a diquark formed by five sets of Dirac matrices, $1, \gamma_{5}, \gamma_{\mu}, \gamma_{\mu} \gamma_{5}$ and $\sigma_{\mu \nu}$

$$
\begin{aligned}
& \Lambda_{1}=\epsilon_{a b c} \epsilon^{A B C}\left(q_{A}^{a T} C q_{B}^{b}\right) \gamma_{5} q_{C}^{c}, \\
& \Lambda_{2}=\epsilon_{a b c} \epsilon^{A B C}\left(q_{A}^{a T} C \gamma_{5} q_{B}^{b}\right) q_{C}^{c}, \\
& \Lambda_{3}=\epsilon_{a b c} \epsilon^{A B C}\left(q_{A}^{a T} C \gamma_{\mu} \gamma_{5} q_{B}^{b}\right) \gamma^{\mu} q_{C}^{c}, \\
& \Lambda_{4}=\epsilon_{a b c} \epsilon^{A B C}\left(q_{A}^{a T} C \gamma_{\mu} q_{B}^{b}\right) \gamma^{\mu} \gamma_{5} q_{C}^{c}, \\
& \Lambda_{5}=\epsilon_{a b c} \epsilon^{A B C}\left(q_{A}^{a T} C \sigma_{\mu \nu} q_{B}^{b}\right) \sigma_{\mu \nu} \gamma_{5} q_{C}^{c} .
\end{aligned}
$$

Among these five fields, we can show that the fourth and fifth ones vanish, $\Lambda_{4,5}=0$. This is due to the Pauli principle between the first two quarks, and can be verified, for instance, by taking the transpose of the diquark component and compare the resulting three-quark field with the original expressions [26]. The Pauli principle can also be used between the first and the third quarks, so we construct the primed fields where the second and the third quarks are interchanged, for instance,

$$
\Lambda_{1}^{\prime}=\epsilon_{a b c} \epsilon^{A B C}\left(q_{A}^{a T} C q_{C}^{c}\right) \gamma_{5} q_{B}^{b}
$$

Now expressing $\Lambda_{i}$ in terms of the Fierz transformed fields $\Lambda_{i}^{\prime}$, we find the following relations (see Appendix C),

$$
\begin{aligned}
& \Lambda_{1}=-\frac{1}{4} \Lambda_{1}^{\prime}-\frac{1}{4} \Lambda_{2}^{\prime}-\frac{1}{4} \Lambda_{3}^{\prime} \\
& \Lambda_{2}=-\frac{1}{4} \Lambda_{1}^{\prime}-\frac{1}{4} \Lambda_{2}^{\prime}+\frac{1}{4} \Lambda_{3}^{\prime} \\
& \Lambda_{3}=-\Lambda_{1}^{\prime}+\Lambda_{2}^{\prime}+\frac{1}{2} \Lambda_{3}^{\prime} .
\end{aligned}
$$

On the other hand, by changing the indices $B, C$ and $b, c$, for instance,

$$
\begin{aligned}
\Lambda_{1}^{\prime} & =\epsilon_{a c b} \epsilon^{A C B}\left(q_{A}^{a T} C q_{B}^{b}\right) \gamma_{5} q_{C}^{c} \\
& =\epsilon_{a b c} \epsilon^{A B C}\left(q_{A}^{a T} C q_{B}^{b}\right) \gamma_{5} q_{C}^{c},
\end{aligned}
$$


we see that the primed fields are just the corresponding unprimed ones, $\Lambda_{i}^{\prime}=\Lambda_{i}$. Consequently, we obtain three homogeneous linear equations whose rank is just one, and we find the following solution

$$
\Lambda_{3}=4 \Lambda_{2}=-4 \Lambda_{1}, \Lambda_{4}=\Lambda_{5}=0
$$

We see that there is only one non-vanishing independent field, which in the quark model corresponds to the odd-parity $\Lambda(1405)$.

\section{The flavour decuplet baryons}

Among the five decuplet baryon fields formed by the five different $\gamma$-matrices, only two are non-zero:

$$
\begin{aligned}
& \Delta_{4}^{P}=\epsilon_{a b c} S_{P}^{A B C}\left(q_{A}^{a T} C \gamma_{\mu} q_{B}^{b}\right) \gamma^{\mu} \gamma_{5} q_{C}^{c}, \\
& \Delta_{5}^{P}=\epsilon_{a b c} S_{P}^{A B C}\left(q_{A}^{a T} C \sigma_{\mu \nu} q_{B}^{b}\right) \sigma_{\mu \nu} \gamma_{5} q_{C}^{c} .
\end{aligned}
$$

Performing the Fierz transformation and with the relation $\Delta_{i}^{P \prime}=-\Delta_{i}^{P}\left(\epsilon_{a c b} S_{P}^{A C B}=-\epsilon_{a b c} S_{P}^{A B C}\right)$, we find that there is only a trivial (null) solution to the homogeneous linear equations. Therefore, the Dirac baryon fields (fundamental representation of the Lorentz group) formed by three quarks can not survive the flavor decuplet.

\section{The flavor octet baryon fields}

Let us start once again with five fields, which have three potentially non-zero ones

$$
\begin{aligned}
& N_{1}^{N}=\epsilon_{a b c} \epsilon^{A B D} \lambda_{D C}^{N}\left(q_{A}^{a T} C q_{B}^{b}\right) \gamma_{5} q_{C}^{c}, \\
& N_{2}^{N}=\epsilon_{a b c} \epsilon^{A B D} \lambda_{D C}^{N}\left(q_{A}^{a T} C \gamma_{5} q_{B}^{b}\right) q_{C}^{c}, \\
& N_{3}^{N}=\epsilon_{a b c} \epsilon^{A B D} \lambda_{D C}^{N}\left(q_{A}^{a T} C \gamma_{\mu} \gamma_{5} q_{B}^{b}\right) \gamma^{\mu} q_{C}^{c}, \\
& N_{4}^{N}=\epsilon_{a b c} \epsilon^{A B D} \lambda_{D C}^{N}\left(q_{A}^{a T} C \gamma_{\mu} q_{B}^{b}\right) \gamma^{\mu} \gamma_{5} q_{C}^{c}=0, \\
& N_{5}^{N}=\epsilon_{a b c} \epsilon^{A B D} \lambda_{D C}^{N}\left(q_{A}^{a T} C \sigma_{\mu \nu} q_{B}^{b}\right) \sigma_{\mu \nu} \gamma_{5} q_{C}^{c}=0 .
\end{aligned}
$$

These octet baryon fields have been studied in Refs [13, 14, 15], where the independent ones are clarified. As before, we perform the Fierz rearrangement to obtain five equations with the primed fields, while $N_{4}^{N \prime}$ and $N_{5}^{N \prime}$ are not zero. For the first three equations, $N_{1,2,3}$ on the left hand side should be expressed by the primed fields. To this end, we can use the Jacobi identity

$$
\epsilon^{A B D} \lambda_{D C}^{N}+\epsilon^{B C D} \lambda_{D A}^{N}+\epsilon^{C A D} \lambda_{D B}^{N}=0
$$

which can be used to relate the original fields $N_{i}^{N}$ and primed ones $N_{i}^{N \prime}$, for instance,

$$
\left(\epsilon^{A B D} \lambda_{D C}^{N}+\epsilon^{B C D} \lambda_{D A}^{N}+\epsilon^{C A D} \lambda_{D B}^{N}\right)\left(q_{A}^{a T} C q_{B}^{b}\right) \gamma_{5} q_{C}^{c}=0
$$

from which we find

$$
N_{1}^{N \prime}=-\frac{1}{2} N_{1}^{N}
$$

and the same relations for $N_{2,3}^{N}$. There are no relations between $N_{4,5}^{N}$ and $N_{4,5}^{N \prime}$. Altogether, we have five equations. The equations related to $N_{4}^{N}$ and $N_{5}^{N}$ are also necessary because the corresponding primed ones are not zero. They can be solved to obtain the following solutions:

$$
\frac{2}{3} N_{4}^{N \prime}=N_{3}^{N}=N_{1}^{N}-N_{2}^{N}, N_{5}^{N \prime}=-3\left(N_{1}^{N}+N_{2}^{N}\right),
$$

which indicates that there are two independent octet fields, for instance, $N_{1}^{N}$ and $N_{2}^{N}$. Thus we have shown the same result just as in the two-flavour case [12]. In the following sections we shall show that the difference between the two fields $N_{1}$ and $N_{2}$ lies in their chiral properties: $N_{1}^{N}-N_{2}^{N}$ together with $\Lambda$ belong to $(\overline{\mathbf{3}}, \mathbf{3}) \oplus(\mathbf{3}, \overline{\mathbf{3}})$, and the other $N_{1}^{N}+N_{2}^{N}$ belongs to $(\mathbf{8}, \mathbf{1}) \oplus(\mathbf{1}, \mathbf{8})$.

There are two ways to construct the octet baryon fields. One is done already as shown in Eqs. (16), whose flavor structure is the same as the $\rho$ type baryon field $N_{8 \rho}^{N}$ in Eqs. (9):

$$
\mathbf{3} \otimes \mathbf{3} \otimes \mathbf{3} \longrightarrow(\mathbf{3} \otimes \mathbf{3}) \otimes \mathbf{3} \longrightarrow \overline{\mathbf{3}} \otimes \mathbf{3} \longrightarrow \mathbf{8}_{\rho} .
$$


The other $\lambda$ type baryon field $N_{8 \lambda}^{N}$ is complicated when used straightforwardly:

$$
3 \otimes 3 \otimes 3 \longrightarrow(3 \otimes 3) \otimes 3 \longrightarrow 6 \otimes 3 \longrightarrow 8_{\lambda} .
$$

Therefore, we use another way based on

$$
\mathbf{3} \otimes \mathbf{3} \otimes \mathbf{3} \longrightarrow \mathbf{3} \otimes(\mathbf{3} \otimes \mathbf{3}) \longrightarrow \mathbf{3} \otimes \overline{\mathbf{3}} \longrightarrow \mathbf{8}_{\rho}^{\prime} .
$$

This contains partly $\mathbf{8}_{\lambda}$, and it is easily to verify that (18) and (20) compose a full description of octet baryon which is also fully described by using (18) and (19). The way $\boldsymbol{8}_{\rho}$ leads to octet fields $N_{i}^{N}$, and the other way $\boldsymbol{8}_{\rho}^{\prime}$ leads to other five ones

$$
\begin{aligned}
& \widetilde{N}_{1}^{N}=\epsilon_{a b c} \epsilon^{A C D} \lambda_{D B}^{N}\left(q_{A}^{a T} C q_{B}^{b}\right) \gamma_{5} q_{C}^{c}, \\
& \widetilde{N}_{2}^{N}=\epsilon_{a b c} \epsilon^{A C D} \lambda_{D B}^{N}\left(q_{A}^{a T} C \gamma_{5} q_{B}^{b}\right) q_{C}^{c}, \\
& \widetilde{N}_{3}^{N}=\epsilon_{a b c} \epsilon^{A C D} \lambda_{D B}^{N}\left(q_{A}^{a T} C \gamma_{\mu} \gamma_{5} q_{B}^{b}\right) \gamma^{\mu} q_{C}^{c}, \\
& \widetilde{N}_{4}^{N}=\epsilon_{a b c} \epsilon^{A C D} \lambda_{D B}^{N}\left(q_{A}^{a T} C \gamma_{\mu} q_{B}^{b}\right) \gamma^{\mu} \gamma_{5} q_{C}^{c}, \\
& \widetilde{N}_{5}^{N}=\epsilon_{a b c} \epsilon^{A C D} \lambda_{D B}^{N}\left(q_{A}^{a T} C \sigma_{\mu \nu} q_{B}^{b}\right) \sigma_{\mu \nu} \gamma_{5} q_{C}^{c} .
\end{aligned}
$$

However, these fields can be related to the previous ones by changing the flavor and color indices $B, C$ and $b, c$ :

$$
\widetilde{N}_{i}^{N}=-N_{i}^{N \prime} .
$$

In nearly all the cases, the octet baryon fields from the second way can be related to the ones from the first way. Therefore, we shall omit the discussion of the second octet. One exception which concerns the chiral representation $(\overline{\mathbf{3}}, \mathbf{3}) \otimes(\mathbf{6}, \mathbf{3})$ is discussed in Appendix D.

\section{A short summary of independent baryon fields}

Properties of baryons fields expressed by the Rarita-Schwinger fields with one Lorentz index and those of the antisymmetric tensor-spinor fields with two Lorentz indices are discussed in Appendices A and B] respectively. Here let us make a short summary for independent baryon fields for all cases constructed by three quarks. For simplicity, here we suppress the antisymmetric tensor in color space $\epsilon_{a b c}$, since it appears in all baryon fields in the same manner. Furthermore, it is convenient to introduce a "tilde-transposed" quark field $\widetilde{q}$ as follows

$$
\widetilde{q}=q^{T} C \gamma_{5} .
$$

As we have shown already, for Dirac fields without Lorentz index, there are one singlet field $\Lambda$ and two octet fields $N_{1}^{N}$ and $N_{2}^{N}$ :

$$
\begin{aligned}
\Lambda_{1} & =\epsilon^{A B C}\left(\widetilde{q}_{A} \gamma_{5} q_{B}\right) \gamma_{5} q_{C}, \\
N_{1}^{N} & =\epsilon^{A B D} \lambda_{D C}^{N}\left(\widetilde{q}_{A} \gamma_{5} q_{B}\right) \gamma_{5} q_{C}, \\
N_{2}^{N} & =\epsilon^{A B D} \lambda_{D C}^{N}\left(\widetilde{q}_{A} q_{B}\right) q_{C} .
\end{aligned}
$$

For the Rarita-Schwinger fields with one Lorentz index, we would consider one singlet, three octet and one decuplet fields:

$$
\begin{aligned}
\Lambda_{1 \mu} & =\epsilon^{A B C}\left(\widetilde{q}_{A} \gamma_{5} q_{B}\right) \gamma_{\mu} q_{C}, \\
N_{1 \mu}^{N} & =\epsilon^{A B D} \lambda_{D C}^{N}\left(\widetilde{q}_{A} \gamma_{5} q_{B}\right) \gamma_{\mu} q_{C}, \\
N_{2 \mu}^{N} & =\epsilon^{A B D} \lambda_{D C}^{N}\left(\widetilde{q}_{A} q_{B}\right) \gamma_{\mu} \gamma_{5} q_{C}, \\
N_{3 \mu}^{N} & =-\epsilon^{A B D} \lambda_{D C}^{N}\left(\widetilde{q}_{A} \gamma_{\mu} q_{B}\right) \gamma_{5} q_{C}, \\
\Delta_{5 \mu}^{P} & =-S_{P}^{A B C}\left(\widetilde{q}_{A} \gamma_{\mu} \gamma_{5} q_{B}\right) q_{C} .
\end{aligned}
$$

However, we find that $\Lambda_{1 \mu}=\gamma_{\mu} \gamma_{5} \Lambda, N_{1 \mu}^{N}=\gamma_{\mu} \gamma_{5} N_{1}^{N}$ and $N_{2 \mu}^{N}=\gamma_{\mu} \gamma_{5} N_{2}^{N}$. So, there are two non-vanishing independent fields: one octet field $N_{\mu}^{N}$ and one decuplet field $\Delta_{\mu}$. By using the projection operator:

$$
P_{\mu \nu}^{3 / 2}=\left(g_{\mu \nu}-\frac{1}{4} \gamma_{\mu} \gamma_{\nu}\right)
$$


they can be written as

$$
\begin{aligned}
N_{\mu}^{N}=P_{\mu \nu}^{3 / 2} N_{3 \nu}^{N} & =-\left(g_{\mu \nu}-\frac{1}{4} \gamma_{\mu} \gamma_{\nu}\right) \epsilon^{A B D} \lambda_{D C}^{N}\left(\widetilde{q}_{A} \gamma_{\mu} q_{B}\right) \gamma_{5} q_{C} \\
& =N_{3 \mu}^{N}+\frac{1}{4} \gamma_{\mu} \gamma_{5}\left(N_{1}^{N}-N_{2}^{N}\right) \\
\Delta_{\mu}^{P}=P_{\mu \nu}^{3 / 2} \Delta_{5 \nu}^{P} & =-\left(g_{\mu \nu}-\frac{1}{4} \gamma_{\mu} \gamma_{\nu}\right) S_{P}^{A B C}\left(\widetilde{q}_{A} \gamma_{\mu} \gamma_{5} q_{B}\right) q_{C} \\
& =\Delta_{5 \mu}^{P}
\end{aligned}
$$

For tensor fields with two antisymmetric Lorentz indices, we would have one singlet, three octet and two decuplet fields:

$$
\begin{aligned}
\Lambda_{1 \mu} & =\epsilon^{A B C}\left(\widetilde{q}_{A} \gamma_{5} q_{B}\right) \sigma_{\mu \nu} \gamma_{5} q_{C}, \\
N_{3 \mu \nu}^{N} & =-\epsilon^{A B D} \lambda_{D C}^{N}\left(\widetilde{q}_{A} \gamma_{\mu} q_{B}\right) \gamma_{\nu} q_{C}+(\mu \leftrightarrow \nu), \\
N_{10 \mu \nu}^{N} & =\epsilon^{A B D} \lambda_{D C}^{N}\left(\widetilde{q}_{A} \gamma_{5} q_{B}\right) \sigma_{\mu \nu} \gamma_{5} q_{C}, \\
N_{11 \mu \nu}^{N} & =\epsilon^{A B D} \lambda_{D C}^{N}\left(\widetilde{q}_{A} q_{B}\right) \sigma_{\mu \nu} q_{C}, \\
\Delta_{2 \mu \nu}^{P} & =-S_{P}^{A B C}\left(\widetilde{q}_{A} \gamma_{\mu} \gamma_{5} q_{B}\right) \gamma_{\nu} \gamma_{5} q_{C}+(\mu \leftrightarrow \nu), \\
\Delta_{7 \mu \nu}^{P} & =S_{P}^{A B C}\left(\widetilde{q}_{A} \sigma_{\mu \nu} \gamma_{5} q_{B}\right) \gamma_{5} q_{C} .
\end{aligned}
$$

But in this case, we can show that there is only one non-vanishing field $\Delta_{\mu \nu}$ :

$$
\begin{aligned}
\Delta_{\mu \nu}^{P}=\Gamma^{\mu \nu \alpha \beta} \Delta_{7 \mu \nu}^{P} & =\Gamma^{\mu \nu \alpha \beta} S_{P}^{A B C}\left(\widetilde{q}_{A} \sigma_{\mu \nu} \gamma_{5} q_{B}\right) \gamma_{5} q_{C} \\
& =\Delta_{7 \mu \nu}^{P}-\frac{i}{2} \gamma_{\mu} \gamma_{5} \Delta_{5 \nu}^{P}+\frac{i}{2} \gamma_{\nu} \gamma_{5} \Delta_{5 \mu}^{P}
\end{aligned}
$$

where

$$
\Gamma^{\mu \nu \alpha \beta}=\left(g^{\mu \alpha} g^{\nu \beta}-\frac{1}{2} g^{\nu \beta} \gamma^{\mu} \gamma^{\alpha}+\frac{1}{2} g^{\mu \beta} \gamma^{\nu} \gamma^{\alpha}+\frac{1}{6} \sigma^{\mu \nu} \sigma^{\alpha \beta}\right)
$$

\section{CHIRAL TRANSFORMATIONS}

In this section, we establish the chiral transformation properties of the baryon fields which we have obtained in the previous section. Technically, this requires somewhat complicated algebra. However, the final result will be understood by making the left and right handed decomposition, as we will perform in the next section.

Let us start with the chiral transformation properties of quarks which are given by the following equations:

$$
\begin{gathered}
\mathbf{U}(\mathbf{1})_{\mathbf{V}}: q \rightarrow \exp \left(i \frac{\lambda^{0}}{2} a_{0}\right) q=q+\delta q, \\
\mathbf{S U}(\mathbf{3})_{\mathbf{V}}: q \rightarrow \exp \left(i \frac{\vec{\lambda}}{2} \vec{a}\right) q=q+\delta^{\vec{a}} q, \\
\mathbf{U}(\mathbf{1})_{\mathbf{A}}: q \rightarrow \exp \left(i \gamma_{5} \frac{\lambda^{0}}{2} b_{0}\right) q=q+\delta_{5} q, \\
\mathbf{S U}(\mathbf{3})_{\mathbf{A}}: q \rightarrow \exp \left(i \gamma_{5} \frac{\vec{\lambda}}{2} \vec{b}\right) q=q+\delta_{5}^{\vec{b}} q,
\end{gathered}
$$

where $\lambda^{0}=\sqrt{2 / 3} 1, a^{0}$ is an infinitesimal parameter for the $U(1)_{V}$ transformation, $\vec{a}$ the octet of $S U(3)_{V}$ group parameters, $b^{0}$ an infinitesimal parameter for the $U(1)_{A}$ transformation, and $\vec{b}$ the octet of the chiral transformations.

The $U(1)_{V}$ chiral transformation is trivial which picks up a phase factor proportional to the baryon number. The 
$U(1)_{A}$ chiral transformation is slightly less trivial, and the baryon fields are transformed as

$$
\begin{aligned}
\delta_{5} \Lambda & =-i \gamma_{5} \sqrt{\frac{1}{6}} b^{0} \Lambda \\
\delta_{5}\left(N_{1}^{N}-N_{2}^{N}\right) & =-i \gamma_{5} \sqrt{\frac{1}{6}} b^{0}\left(N_{1}^{N}-N_{2}^{N}\right), \\
\delta_{5}\left(N_{1}^{N}+N_{2}^{N}\right) & =i \gamma_{5} \sqrt{\frac{3}{2}} b^{0}\left(N_{1}^{N}+N_{2}^{N}\right), \\
\delta_{5} N_{\mu}^{N} & =i \gamma_{5} \sqrt{\frac{1}{6}} b^{0} N_{\mu}^{N} \\
\delta_{5} \Delta_{\mu}^{P} & =i \gamma_{5} \sqrt{\frac{1}{6}} b^{0} \Delta_{\mu}^{P} \\
\delta_{5} \Delta_{\mu \nu}^{P} & =i \gamma_{5} \sqrt{\frac{3}{2}} b^{0} \Delta_{\mu \nu}^{P} .
\end{aligned}
$$

We note that the combinations of $N_{1}^{N} \pm N_{2}^{N}$ form different representations.

Under the vector chiral transformation, the baryon fields are transformed as

$$
\begin{aligned}
\delta^{\vec{a}} \Lambda & =0, \\
\delta^{\vec{a}} N_{1}^{N} & =-a^{M} f^{N M O} N_{1}^{O}, \\
\delta^{\vec{a}} N_{2}^{N} & =-a^{M} f^{N M O} N_{2}^{O}, \\
\delta^{\vec{a}} N_{\mu}^{N} & =-a^{M} f^{N M O} N_{\mu}^{N}, \\
\delta^{\vec{a}} \Delta_{\mu}^{P} & =\frac{3 i}{2} a^{M} g_{7}^{P M Q} \Delta_{\mu}^{Q}, \\
\delta^{\vec{a}} \Delta_{\mu \nu}^{P} & =\frac{3 i}{2} a^{M} g_{7}^{P M Q} \Delta_{\mu \nu}^{Q},
\end{aligned}
$$

where $f^{A B C}$ is the standard antisymmetric structure constant of $S U(3)$, and $g_{7}^{A B C}$ is defined in Table $\amalg$ Eqs. (27) show nothing but the flavor charge of the baryons. For example, we can show explicitly:

$$
\delta^{a 3} p=+\frac{i}{2} a_{3} p, \delta^{a 3} n=-\frac{i}{2} a_{3} n, \delta^{a 3} \Delta^{++}=\frac{3 i}{2} a_{3} \Delta^{++} \ldots
$$

The transformation rule under the axial-vector chiral transformations are rather complicated as they are no longer conserved and reflect the internal structure of baryons. To start with, we have the axial transformation of the three-quark baryon fields such as

$$
\delta_{5}^{\vec{b}} \Lambda=\epsilon_{a b c} \epsilon^{A B C}\left(\left(q_{A}^{a T} C q_{B}^{b}\right) \gamma_{5}\left(\delta_{5}^{\vec{b}} q_{C}^{c}\right)+\left(q_{A}^{a T} C\left(\delta_{5}^{\vec{b}} q_{B}^{b}\right)\right) \gamma_{5} q_{C}^{c}+\left(\left(\delta_{5}^{\vec{b}} q_{A}^{a T}\right) C q_{B}^{b}\right) \gamma_{5} q_{C}^{c}\right) .
$$

The calculation is complicated, but rather straightforward. Here, we show therefore the final result of the axial transformation:

$$
\begin{aligned}
\delta_{5}^{\vec{b}} \Lambda & =\frac{i}{2} \gamma_{5} b^{N}\left(N_{1}^{N}-N_{2}^{N}\right), \\
\delta_{5}^{\vec{b}}\left(N_{1}^{N}-N_{2}^{N}\right) & =\frac{4 i}{3} \gamma_{5} b^{N} \Lambda+i \gamma_{5} b^{M} d^{N M O}\left(N_{1}^{O}-N_{2}^{O}\right), \\
\delta_{5}^{\vec{b}}\left(N_{1}^{N}+N_{2}^{N}\right) & =-\gamma_{5} b^{M} f^{N M O}\left(N_{1}^{O}+N_{2}^{O}\right), \\
\delta_{5}^{\vec{b}} N_{\mu}^{N} & =i \gamma_{5} b^{M}\left(d^{M N O}-\frac{2 i}{3} f^{M N O}\right) N_{\mu}^{O}+i \gamma_{5} b^{M} g_{3}^{M N P} \Delta_{\mu}^{P}, \\
\delta_{5}^{\vec{b}} \Delta_{\mu}^{P} & =-2 i \gamma_{5} b^{M} g_{5}^{P M O} N_{\mu}^{O}+\frac{i}{2} \gamma_{5} b^{M} g_{7}^{P M Q} \Delta_{\mu}^{Q}, \\
\delta_{5}^{\vec{b}} \Delta_{\mu \nu}^{P} & =\frac{3 i}{2} \gamma_{5} b^{M} g_{7}^{P M Q} \Delta_{\mu \nu}^{Q} .
\end{aligned}
$$


The coefficients $d^{A B C}$ are the standard symmetric structure constants of $S U(3)$. For completeness, we show the following equation which define the $d$ and $f$ coefficients

$$
\begin{aligned}
\lambda_{A B}^{N} \lambda_{B C}^{M} & =\left(\lambda^{N} \lambda^{M}\right)_{A C}=\frac{1}{2}\left\{\lambda^{N}, \lambda^{M}\right\}_{A C}+\frac{1}{2}\left[\lambda^{N}, \lambda^{M}\right]_{A C} \\
& =\frac{2}{3} \delta^{N M} \delta_{A C}+\left(d^{N M O}+i f^{N M O}\right) \lambda_{A C}^{O} .
\end{aligned}
$$

Furthermore, the following formulae define the coefficients $g_{3}, g_{5}$ and $g_{7}$, which are proved by using Mathematica, a software good at matrix calculation:

$$
\begin{aligned}
& \epsilon^{A D E} \lambda_{D B}^{N} \lambda_{E C}^{M}=g_{1}^{N M O} \epsilon^{A B D} \lambda_{D C}^{O}+g_{2}^{N M O} \epsilon^{A C D} \lambda_{D B}^{O}+g_{3}^{N M P} S_{P}^{A B C}+g_{4}^{N M} \epsilon^{A B C} \\
& S_{Q}^{A B D} \lambda_{D C}^{M}=g_{5}^{Q M O} \epsilon^{A B D} \lambda_{D C}^{O}+g_{6}^{Q M O} \epsilon^{A C D} \lambda_{D B}^{O}+g_{7}^{Q M P} S_{P}^{A B C}+g_{8}^{Q M} \epsilon^{A B C}
\end{aligned}
$$

where indices $A \sim E$ take values 1,2 and $3, N, M$ and $O 1, \cdots, 8$, and $P$ and $Q 1, \cdots, 10$. The coefficients $g_{3}, g_{5}$ and $g_{7}$ are listed in Table $\amalg$ where we use "0" instead of " 10 ". Other coefficients can be related to $d, f, g_{3}, g_{5}$ and $g_{7}$ :

$$
\begin{aligned}
g_{1}^{M N O} & =-d^{M N O}-\frac{i}{3} f^{M N O}, \\
g_{2}^{M N O} & =d^{M N O}-\frac{i}{3} f^{M N O}, \\
g_{4}^{M N} & =-\frac{1}{3} \delta^{M N}, \\
g_{6}^{Q M O} & =-2 g_{5}^{Q M O}, \\
g_{8}^{M N} & =0 .
\end{aligned}
$$

Let us explain Eqs. (30) a bit more. The quantities on the left hand side have three indices $A, B$ and $C$, and therefore, they are regarded as direct products of three fundamental representations of $S U(3): \mathbf{3} \otimes \mathbf{3} \otimes \mathbf{3}$. They can be decomposed into irreducible components by applying the four kinds of operators: $\epsilon_{A B C}, \epsilon^{A B D} \lambda_{D C}^{O}, \epsilon^{A C D} \lambda_{D B}^{O}$ and $S_{P}^{A B C}$, which correspond to $\mathbf{1}, \mathbf{8}, \mathbf{8}$ and $\mathbf{1 0}$ of $S U(3)$, respectively.

Eqs. (26) and (28) imply that $\Lambda$ and $N_{1}^{N}-N_{2}^{N}$ are together combined into one chiral multiplet, and $N_{\mu}^{N}$ and $\Delta_{\mu}^{P}$ are together combined into another chiral multiplet. While $N_{1}^{N}+N_{2}^{N}$ and $\Delta_{\mu \nu}^{P}$ are transformed into themselves under chiral transformation. In our following discussion, we will find that $\Lambda$ and $N_{1}^{N}-N_{2}^{N}$ belong to the chiral representation $(\overline{\mathbf{3}}, \mathbf{3}) \oplus(\mathbf{3}, \overline{\mathbf{3}}), N_{1}^{N}+N_{2}^{N}$ belongs to the chiral representation $(\mathbf{8}, \mathbf{1}) \oplus(\mathbf{1}, \mathbf{8}), N_{\mu}^{N}$ and $\Delta_{\mu}^{P}$ belong to the chiral representation $(\mathbf{6}, \mathbf{3}) \oplus(\mathbf{3}, \mathbf{6})$, and $\Delta_{\mu \nu}^{P}$ belongs to the chiral representation $(\mathbf{1 0}, \mathbf{1}) \oplus(\mathbf{1}, \mathbf{1 0})$. We show several examples of the axial-vector chiral transformation:

$$
\delta_{5}^{b 3} p_{-}=\frac{i}{2} \gamma_{5} b_{3} p_{-}, \delta_{5}^{b 3} p_{+}=\frac{i}{2} \gamma_{5} b_{3} p_{+}, \delta_{5}^{b 3} p_{\mu}=\frac{5 i}{6} \gamma_{5} b_{3} p_{\mu}-\frac{4 i}{3} \gamma_{5} b_{3} \Delta_{\mu}^{+}
$$

where $p_{-}$belongs to the octet baryon fields $N_{1}^{N}-N_{2}^{N}, p_{+}$belongs to $N_{1}^{N}+N_{2}^{N}$, and $p_{\mu}$ belongs to $N_{\mu}^{N}$ (see Eqs. (11)).

\section{CHIRAL MULTIPLETS/REPRESENTATIONS}

So far, we have performed classifications without explicitly taking into account the left- and right-handed components of the quark fields. However, it does not require great imagination to see that the chiral properties are also conveniently studied in that language, since chiral symmetry is defined as the symmetries upon each chiral field. Hence, we define the left- and right-handed (chiral or Weyl representation) quark fields as

$$
L \equiv q_{L}=\frac{1-\gamma_{5}}{2} q, \quad \text { and } \quad R \equiv q_{R}=\frac{1+\gamma_{5}}{2} q .
$$

They form the fundamental representations of both the Lorentz group and the chiral group,

$$
\begin{array}{lll}
L: & \text { Lorentz }:\left(\frac{1}{2}, 0\right), & \text { Chiral }:(3,1), \\
R: & \text { Lorentz }:\left(0, \frac{1}{2}\right), & \text { Chiral }:(1,3) .
\end{array}
$$


TABLE II: $g$-coefficients defined by Eqs. (30)

\begin{tabular}{|c|c|c|c|c|c|c|c|}
\hline \multirow[t]{8}{*}{$g_{3}$} & \multicolumn{6}{|c|}{$133,138,144,146,254,256,272,279,439,463,468,573,578,612,619,636$} & $-1 / 3$ \\
\hline & \multicolumn{6}{|c|}{$162,169,313,318,349,366,414,416,524,526,643,648,722,729,753,758$} & $1 / 3$ \\
\hline & \multicolumn{6}{|c|}{$154,179,215,233,246,269,328,359,376,424,455,478,516,563,622,658,712,743,765$} & $-i / 3$ \\
\hline & \multicolumn{6}{|c|}{$125,156,172,238,244,262,323,426,473,514,539,545,568,629,653,675,719,736,748$} & $i / 3$ \\
\hline & $183,686,818,835,849$ & \multicolumn{4}{|c|}{\begin{tabular}{|l|l|l|l|}
$-1 / \sqrt{3}$ & $167,251,277,411,570,640$ & -1 \\
\end{tabular}} & 342,364 & $-2 / 3$ \\
\hline & $188,385,489,813,866$ & $1 / \sqrt{3}$ & \multicolumn{2}{|c|}{$141,460,521,617,727,750$} & 1 & 432,634 & $2 / 3$ \\
\hline & $283,288,589,876$ & $-i / \sqrt{3}$ & \multicolumn{2}{|c|}{$177,421,470,511,560,627$} & $-i$ & 352,374 & $-2 i / 3$ \\
\hline & $786,823,828,859$ & $i / \sqrt{3}$ & \multicolumn{2}{|c|}{$151,241,267,650,717,740$} & $i$ & 532,734 & $2 i / 3$ \\
\hline \multirow[t]{9}{*}{$g_{5}$} & \multirow{2}{*}{\multicolumn{3}{|c|}{$\begin{array}{l}125,141,227,261,313,346,357,414,425,614,625 \\
663,716,727,813,846,857,927,943,961,057,064 \\
\end{array}$}} & \multirow[t]{2}{*}{$1 / 6$} & & $318,668,881,984$ & $1 / 2 \sqrt{3}$ \\
\hline & & & & & & $381,686,818,948$ & $-1 / 2 \sqrt{3}$ \\
\hline & \multirow{2}{*}{\multicolumn{3}{|c|}{$\begin{array}{l}114,152,216,272,331,364,375,441,452,636,641 \\
652,761,772,831,864,875,916,934,972,046,075 \\
\end{array}$}} & & & $382,678,882,985$ & $i / 2 \sqrt{3}$ \\
\hline & & & & & & $328,687,828,958$ & $-i / 2 \sqrt{3}$ \\
\hline & \multirow{4}{*}{\multicolumn{3}{|c|}{$\begin{array}{l}115,124,217,226,332,347,365,424,451,615,642 \\
673,726,771,823,856,874,953,962,971,065,074 \\
142,151,262,271,323,356,374,415,442,624,637 \\
651,717,762,832,847,865,917,926,935,047,056 \\
\end{array}$}} & \multirow[t]{2}{*}{$i / 6$} & & 234,436 & $1 / 3$ \\
\hline & & & & & & 243,463 & $-1 / 3$ \\
\hline & & & & \multirow[t]{2}{*}{$-i / 6$} & & $53,473,512,554,567$ & $i / 3$ \\
\hline & & & & & & $35,437,521,545,576$ & $-i / 3$ \\
\hline & \multicolumn{3}{|c|}{583} & \multirow{2}{*}{\begin{tabular}{|c|}
$1 / \sqrt{3}$ \\
$1 / 3$
\end{tabular}} & \multicolumn{2}{|r|}{538} & $-1 / \sqrt{3}$ \\
\hline \multirow[t]{8}{*}{$g_{7}$} & \multicolumn{3}{|c|}{$\begin{array}{l}112,143,232,245,263,315,362,448,465,619 \\
636,665,714,768,815,844,916,945,046,069 \\
\end{array}$} & & \multicolumn{2}{|c|}{$\begin{array}{c}214,333,346,412,513,518 \\
542,549,564,566,643,869,968 \\
\end{array}$} & $2 / 3$ \\
\hline & \multicolumn{3}{|c|}{434,939} & $-1 / 3$ & \multicolumn{2}{|r|}{838} & $-2 / 3$ \\
\hline & \multicolumn{3}{|c|}{$372,675,724,825,854,926,955,056,079$} & $i / 3$ & \multicolumn{2}{|c|}{$422,523,552,574,653,978$} & $2 i / 3$ \\
\hline & \multirow{2}{*}{\multicolumn{3}{|c|}{$\frac{122,153,255,273,325,458,475,629,778}{131,211,341,417,640,867,960}$}} & $-i / 3$ & \multicolumn{2}{|c|}{$224,356,528,559,576,879$} & $-2 i / 3$ \\
\hline & \multicolumn{2}{|c|}{$131,211,341,417,640,867,960$} & & 1 & \multicolumn{2}{|c|}{$181,282,484,787$} & $1 / \sqrt{3}$ \\
\hline & & 737 & & -1 & & 686,989 & $-1 / \sqrt{3}$ \\
\hline & 221 , & 351,87 & & $\imath$ & & 080 & $-2 / \sqrt{3}$ \\
\hline & 427, & 0 , & & $-i$ & & & \\
\hline
\end{tabular}

It is convenient first to note that $\gamma$-matrices are classified into two categories; chiral-even and chiral-odd classes. The chiral-even $\gamma$-matrices survive forming diquarks with identical chiralities, while the chiral-odd ones form diquarks from quarks with opposite chiralities. The chiral-even and -odd $\gamma$-matrices are

$$
\begin{array}{cl}
\text { chiral-even: } & 1, \gamma_{5}, \sigma_{\mu \nu}, \\
\text { chiral-odd: } & \gamma_{\mu}, \gamma_{\mu} \gamma_{5} .
\end{array}
$$

Therefore, we have six non-vanishing diquarks in the chiral representations,

$$
\left.\begin{array}{r}
L^{T} C L=-L^{T} C \gamma_{5} L \\
R^{T} C R=+R^{T} C \gamma_{5} R
\end{array}\right\} \quad(0,0) \oplus(0,0), \quad(\overline{\mathbf{3}}, \mathbf{1}) \oplus(\mathbf{1}, \overline{\mathbf{3}}),
$$

where we have indicated the Lorentz and chiral representations of the diquarks.

For three quarks, we have

$$
(L+R)^{3} \rightarrow\left\{\begin{array}{lll}
L L L & \left(\frac{1}{2}, 0\right) \oplus\left(\frac{3}{2}, 0\right), & (\mathbf{1}, \mathbf{1}) \oplus(\mathbf{8}, \mathbf{1}) \oplus(\mathbf{8}, \mathbf{1}) \oplus(\mathbf{1 0}, \mathbf{1}) \\
L L R & \left(0, \frac{1}{2}\right) \oplus\left(1, \frac{1}{2}\right), & (\overline{\mathbf{3}}, \mathbf{3}) \oplus(\mathbf{6}, \mathbf{3})
\end{array}\right.
$$

and together with the terms where $L$ and $R$ are exchanged. Now we discuss the independent fields in terms of the chiral representations. Once again, for illustration we will discuss here the case of the simplest Lorentz representation $\left(\frac{1}{2}, 0\right) \oplus\left(0, \frac{1}{2}\right)$ for the Dirac fields. 


\section{A. Independent $(L L) L$ fields}

The $(L L) L$ must belong to one of the following chiral representations: $(\mathbf{1}, \mathbf{1}) \oplus(\mathbf{8}, \mathbf{1}) \oplus(\mathbf{8}, \mathbf{1}) \oplus(\mathbf{1 0}, \mathbf{1})$. For each chiral representation, there is one flavor representation available.

For $(\mathbf{1}, \mathbf{1}) \rightarrow \mathbf{1}_{\mathbf{f}}$, there are apparently two non-zero fields

$$
\begin{aligned}
& \Lambda_{L 1}=\epsilon_{a b c} \epsilon^{A B C}\left(L_{A}^{a T} C L_{B}^{b}\right) \gamma_{5} L_{C}^{c}, \\
& \Lambda_{L 2}=\epsilon_{a b c} \epsilon^{A B C}\left(L_{A}^{a T} C \gamma_{5} L_{B}^{b}\right) L_{C}^{c} \\
& \Lambda_{L 3}=\epsilon_{a b c} \epsilon^{A B C}\left(L_{A}^{a T} C \gamma_{\mu} \gamma_{5} L_{B}^{b}\right) \gamma^{\mu} L_{C}^{c}=0,
\end{aligned}
$$

where $\Lambda_{3}^{L}$ vanishes because $\gamma_{\mu} \gamma_{5}$ is chiral-odd

$$
L^{T} C \gamma_{\mu} \gamma_{5} L=0
$$

After performing the Fierz transformation to relate $\Lambda_{L i}$ and $\Lambda_{L i}^{\prime}$ as we have done before, and solving the coupled equations, we find the solution that all such fields vanish.

For $(\mathbf{1 0}, \mathbf{1}) \rightarrow \mathbf{1 0}_{\mathbf{f}}$, we would have again two non-zero components:

$$
\begin{aligned}
& \Delta_{L 4}^{P}=\epsilon_{a b c} S_{P}^{A B C}\left(L_{A}^{a T} C \gamma_{\mu} L_{B}^{b}\right) \gamma^{\mu} \gamma_{5} L_{C}^{c}, \\
& \Delta_{L 5}^{P}=\epsilon_{a b c} S_{P}^{A B C}\left(L_{A}^{a T} C \sigma_{\mu \nu} L_{B}^{b}\right) \sigma^{\mu \nu} \gamma_{5} L_{C}^{c} .
\end{aligned}
$$

Performing the Fierz transformation to relate $\Delta_{L i}^{P}$ and $\Delta_{L i}^{P \prime}$, we obtain the solution that all such $(L L) L$ fields vanish.

Finally for $(\mathbf{8}, \mathbf{1}) \rightarrow \mathbf{8}_{\mathbf{f}}$, we may consider once again two non-zero fields to start with

$$
\begin{aligned}
& N_{L 1}^{N}=\epsilon_{a b c} \epsilon^{A B D} \lambda_{D C}^{N}\left(L_{A}^{a T} C L_{B}^{b}\right) \gamma_{5} L_{C}^{c}, \\
& N_{L 2}^{N}=\epsilon_{a b c} \epsilon^{A B D} \lambda_{D C}^{N}\left(L_{A}^{a T} C \gamma_{5} L_{B}^{b}\right) L_{C}^{c}
\end{aligned}
$$

Applying the Fierz transformation to relate $N_{L i}^{N}$ and $N_{L i}^{N \prime}$, we obtain the solution

$$
N_{L 2}^{N}=N_{L 1}^{N}
$$

Therefore, there is only one independent $(L L) L \mathbf{8}_{\mathbf{f}}$ field.

\section{B. Independent $(L L) R$ fields}

The chiral representations of $(L L) R$ are $(\overline{\mathbf{3}}, \mathbf{3}) \oplus(\mathbf{6}, \mathbf{3})$. We will study them separately in the following.

For $(\overline{\mathbf{3}}, \mathbf{3}) \rightarrow \mathbf{1}_{\mathbf{f}}$, there appears to exist two non-zero components among the five fields,

$$
\begin{aligned}
& \Lambda_{M 1}=\epsilon_{a b c} \epsilon^{A B C}\left(L_{A}^{a T} C L_{B}^{b}\right) \gamma_{5} R_{C}^{c}, \\
& \Lambda_{M 2}=\epsilon_{a b c} \epsilon^{A B C}\left(L_{A}^{a T} C \gamma_{5} L_{B}^{b}\right) R_{C}^{c} \\
& \Lambda_{M 3}=\epsilon_{a b c} \epsilon^{A B C}\left(L_{A}^{a T} C \gamma_{\mu} \gamma_{5} L_{B}^{b}\right) \gamma^{\mu} R_{C}^{c}=0 \\
& \Lambda_{M 4}=\epsilon_{a b c} \epsilon^{A B C}\left(L_{A}^{a T} C \gamma_{\mu} L_{B}^{b}\right) \gamma^{\mu} \gamma_{5} R_{C}^{c}=0, \\
& \Lambda_{M 5}=\epsilon_{a b c} \epsilon^{A B C}\left(L_{A}^{a T} C \sigma_{\mu \nu} L_{B}^{b}\right) \sigma^{\mu \nu} \gamma_{5} R_{C}^{c}=0,
\end{aligned}
$$

where $M$ (mixed) indicates that the fields contain both left and right handed quarks. Performing the Fierz transformation to relate $\Lambda_{M i}$ and $\Lambda_{M i}^{\prime}$, we obtain the following relations

$$
\Lambda_{M 4}^{\prime}=-\Lambda_{M 3}^{\prime}=-2 \Lambda_{M 2}=2 \Lambda_{M 1}
$$

We may consider other ten combinations formed by $(L R)$ and $(R L)$ diquarks, $(L R) L$ and $(R L) L$. However, they can be related to the above ones of $(L L) R$ by a rearrangement of indices as well as the Fierz transformation, for instance,

$$
\Lambda_{M 6}=\epsilon_{a b c} \epsilon^{A B C}\left(L_{A}^{a T} C R_{B}^{b}\right) \gamma_{5} L_{C}^{c}=\Lambda_{M 1}^{\prime}
$$

Therefore, we have only one independent field.

For the chiral representation $(\mathbf{6}, \mathbf{3}) \rightarrow \mathbf{1 0}_{\mathbf{f}}$, we can write five fields containing diquarks formed by five Dirac matrices. However, we can show that after performing the Fierz transformation all fields vanish. Therefore, this representation can not support three-quark fields. 
The baryon fields of chiral representations $(\overline{\mathbf{3}}, \mathbf{3}) \rightarrow \mathbf{8}_{\mathbf{f}}$ can be formed

$$
\begin{aligned}
& N_{M 1}^{N}=\epsilon_{a b c} \epsilon^{A B D} \lambda_{D C}^{N}\left(L_{A}^{a T} C L_{B}^{b}\right) \gamma_{5} R_{C}^{c}, \\
& N_{M 2}^{N}=\epsilon_{a b c} \epsilon^{A B D} \lambda_{D C}^{N}\left(L_{A}^{a T} C \gamma_{5} L_{B}^{b}\right) R_{C}^{c}, \\
& N_{M 3}^{N}=\epsilon_{a b c} \epsilon^{A B D} \lambda_{D C}^{N}\left(L_{A}^{a T} C \gamma_{\mu} \gamma_{5} L_{B}^{b}\right) \gamma^{\mu} R_{C}^{c}=0, \\
& N_{M 4}^{N}=\epsilon_{a b c} \epsilon^{A B D} \lambda_{D C}^{N}\left(L_{A}^{a T} C \gamma_{\mu} L_{B}^{b}\right) \gamma^{\mu} \gamma_{5} R_{C}^{c}=0, \\
& N_{M 5}^{N}=\epsilon_{a b c} \epsilon^{A B D} \lambda_{D C}^{N}\left(L_{A}^{a T} C \sigma_{\mu \nu} L_{B}^{b}\right) \sigma^{\mu \nu} \gamma_{5} R_{C}^{c}=0,
\end{aligned}
$$

where we see that there are two non-zero fields. Applying the Fierz transformation, we can verify that there is only one independent field with the following relations

$$
N_{M 4}^{N \prime}=-N_{M 3}^{N \prime}=-2 N_{M 2}^{N}=2 N_{M 1}^{N}
$$

Another chiral representation $(\mathbf{6}, \mathbf{3}) \rightarrow \mathbf{8}_{\mathbf{f}}$ can be constructed by the combinations similar to (39), for instance,

$$
N_{(6,3) 1}^{N}=\epsilon_{a b c} \epsilon^{A C D} \lambda_{D B}^{N}\left\{\left(L_{A}^{a T} C L_{B}^{b}\right) \gamma_{5} R_{C}^{c}+\left(L_{B}^{a T} C L_{A}^{b}\right) \gamma_{5} R_{C}^{c}\right\}
$$

After similar algebra we can verify that all these fields vanish.

\section{A short summary of chiral representations}

To summarize this section, we find that possible chiral representations for Dirac spinor baryon fields without Lorentz index are:

$$
\begin{aligned}
\Lambda & =\epsilon_{a b c} \epsilon^{A B C}\left(L_{A}^{a T} C L_{B}^{b}\right) \gamma_{5} R_{C}^{c}+\epsilon_{a b c} \epsilon^{A B C}\left(R_{A}^{a T} C R_{B}^{b}\right) \gamma_{5} L_{C}^{c} \\
& =\Lambda_{M 1}+(L \leftrightarrow R), \\
N_{1}^{N}-N_{2}^{N} & =2 \epsilon_{a b c} \epsilon^{A B D} \lambda_{D C}^{N}\left(L_{A}^{a T} C L_{B}^{b}\right) \gamma_{5} R_{C}^{c}+2 \epsilon_{a b c} \epsilon^{A B D} \lambda_{D C}^{N}\left(R_{A}^{a T} C R_{B}^{b}\right) \gamma_{5} L_{C}^{c} \\
& =2 N_{M 1}^{N}+(L \leftrightarrow R), \\
N_{1}^{N}+N_{2}^{N} & =2 \epsilon_{a b c} \epsilon^{A B D} \lambda_{D C}^{N}\left(L_{A}^{a T} C L_{B}^{b}\right) \gamma_{5} L_{C}^{c}+2 \epsilon_{a b c} \epsilon^{A B D} \lambda_{D C}^{N}\left(R_{A}^{a T} C R_{B}^{b}\right) \gamma_{5} R_{C}^{c} \\
& =2 N_{L 1}^{N}+(L \leftrightarrow R) .
\end{aligned}
$$

So we can see that the fields $\Lambda$ and $N_{1}^{N}-N_{2}^{N}$ has a type of $L L R \oplus R R L$, and belong to the chiral representation $(\overline{\mathbf{3}}, \mathbf{3}) \oplus(\mathbf{3}, \overline{\mathbf{3}})$; while the field $N_{1}^{N}+N_{2}^{N}$ has a type of $L L L \oplus R R R$, and belongs to the chiral representation $(\mathbf{8}, \mathbf{1}) \oplus(\mathbf{1}, \mathbf{8})$.

The chiral properties of Rarita-Schwinger fields (Lorentz rep. $\left(\mathbf{1}, \frac{\mathbf{1}}{\mathbf{2}}\right) \oplus\left(\frac{\mathbf{1}}{\mathbf{2}}, \mathbf{1}\right)$ ) are listed in Appendix D. We summarize the results here:

$$
\begin{aligned}
N_{\mu}^{N} & =2 \epsilon_{a b c} \epsilon^{A B D} \lambda_{D C}^{N}\left(L_{A}^{a T} C \gamma_{\mu} \gamma_{5} R_{B}^{b}\right) \gamma_{5} L_{C}^{c}+2 \epsilon_{a b c} \epsilon^{A B D} \lambda_{D C}^{N}\left(R_{A}^{a T} C \gamma_{\mu} \gamma_{5} L_{B}^{b}\right) \gamma_{5} R_{C}^{c} \\
& +\frac{1}{2} \epsilon_{a b c} \epsilon^{A B D} \lambda_{D C}^{N}\left(L_{A}^{a T} C L_{B}^{b}\right) \gamma_{\mu} R_{C}^{c}+\frac{1}{2} \epsilon_{a b c} \epsilon^{A B D} \lambda_{D C}^{N}\left(R_{A}^{a T} C R_{B}^{b}\right) \gamma_{\mu} L_{C}^{c} \\
\Delta_{\mu}^{P} & =2 \epsilon_{a b c} S_{P}^{A B C}\left(L_{A}^{a T} C \gamma_{\mu} R_{B}^{b}\right) L_{C}^{c}+2 \epsilon_{a b c} S_{P}^{A B C}\left(R_{A}^{a T} C \gamma_{\mu} L_{B}^{b}\right) R_{C}^{c} .
\end{aligned}
$$

So we see that $N_{\mu}^{N}$ and $\Delta_{\mu}^{P}$ are of the type $L L R \oplus R R L$, and belong to the chiral representation $(\mathbf{6}, \mathbf{3}) \oplus(\mathbf{3}, \mathbf{6})$. The (similar) results for $\Delta_{\mu \nu}^{P}$, which is of the type $L L L \oplus R R R$, and belongs to the chiral representation $(\mathbf{1 0}, \mathbf{1}) \oplus(\mathbf{1}, \mathbf{1 0})$, are omitted here.

\section{AXIAL COUPLING CONSTANTS}

As a simple application of the present mathematical formalism, we can extract the (diagonal) axial coupling constants $g_{A}$ of baryons. All information is contained in Eqs. (26) and (28), from which we can calculate the Abelian $U(1)_{A}$ axial coupling constant $g_{A}^{0}$ and the non-Abelian $S U(3)_{V} \times S U(3)_{A}$ diagonal axial coupling constants, $g_{A}^{3}$ and $g_{A}^{8}$, which can be extracted from the chiral transformations $\delta_{5}, \delta_{5}^{b 3}$ and $\delta_{5}^{b 8}$, respectively. The Abelian $g_{A}^{0}$ basically counts the difference between the numbers of left- and right- handed quarks in a baryon. In general, diagonal elements of the $S U(3) g_{A}$ 's can be decomposed into $F$ and $D$ components, which are defined by the axial vector current $A_{\mu}^{a}$ $(a=0,1, \ldots 8)$

$$
A_{\mu}^{a}=g_{A}^{F} \operatorname{tr} \overline{\mathfrak{B}} \gamma_{\mu} \gamma_{5}\left[\frac{\lambda_{a}}{2}, \mathfrak{B}\right]+g_{A}^{D} \operatorname{tr} \overline{\mathfrak{B}} \gamma_{\mu} \gamma_{5}\left\{\frac{\lambda_{a}}{2}, \mathfrak{B}\right\}
$$


where $\mathfrak{B}$ is the $3 \times 3$ baryon matrix, Eq. (12). Therefore, we have

$$
\begin{aligned}
A_{\mu}^{3} & =\left(g_{A}^{F}+g_{A}^{D}\right)\left(p^{+} p-n^{+} n\right) \\
& +2 g_{A}^{F}\left(\left(\Sigma^{+}\right)^{+} \Sigma^{+}-\left(\Sigma^{-}\right)^{+} \Sigma^{-}\right) \\
& +\left(g_{A}^{F}-g_{A}^{D}\right)\left(\left(\Xi^{0}\right)^{+} \Xi^{0}-\left(\Xi^{-}\right)^{+} \Xi^{-}\right) \\
A_{\mu}^{8} & =\left(\sqrt{3} g_{A}^{F}-\frac{g_{A}^{D}}{\sqrt{3}}\right)\left(p^{+} p+n^{+} n\right) \\
& +\frac{2 g_{A}^{D}}{\sqrt{3}}\left(\left(\Sigma^{+}\right)^{+} \Sigma^{+}+\left(\Sigma^{-}\right)^{+} \Sigma^{-}\right) \\
& +\left(-\sqrt{3} g_{A}^{F}-\frac{g_{A}^{D}}{\sqrt{3}}\right)\left(\left(\Xi^{0}\right)^{+} \Xi^{0}+\left(\Xi^{-}\right)^{+} \Xi^{-}\right)-\frac{2 g_{A}^{D}}{\sqrt{3}}\left(\Lambda^{8}\right)^{+} \Lambda^{8}
\end{aligned}
$$

where we omit the Lorentz part. In other words,

$$
\begin{aligned}
& g_{A}^{3}(N) \sim\left(g_{A}^{F}+g_{A}^{D}\right) \mathbf{I}_{\mathbf{z}}, \quad g_{A}^{3}(\Sigma) \sim 2 g_{A}^{D} \mathbf{I}_{\mathbf{z}}, \quad g_{A}^{3}(\Xi) \sim\left(g_{A}^{F}-g_{A}^{D}\right) \mathbf{I}_{\mathbf{z}}, \\
& g_{A}^{8}(N) \sim \sqrt{3} g_{A}^{F}-\frac{g_{A}^{D}}{\sqrt{3}}, \quad g_{A}^{8}(\Sigma) \sim \frac{2 g_{A}^{D}}{\sqrt{3}}, \quad g_{A}^{8}(\Xi) \sim-\sqrt{3} g_{A}^{F}-\frac{g_{A}^{D}}{\sqrt{3}}, \quad g_{A}^{8}(\Lambda) \sim-\frac{2 g_{A}^{D}}{\sqrt{3}},
\end{aligned}
$$

for the octet parts. The operator $\mathbf{I}_{\mathbf{z}}$ is the third component of isospin. While the singlet part $g_{A}^{0}$ contains only the $D$ term and is trivial.

For the decuplet baryons, the $S U(3)$ coupling constants contain only one $S U(3)$ irreducible term because the $S U(3)$ Clebsch-Gordan series for $\overline{\mathbf{1 0}} \otimes \mathbf{1 0} \otimes \mathbf{8}$ contains only one singlet. In order to extract the coupling constants, we first rewrite Eqs. (26) and (28) in the following form, for all the singlet, octet and decuplet baryon fields:

1. Because $\lambda_{11}^{0}=\lambda_{22}^{0}=\lambda_{33}^{0}$ for $g_{A}^{0}$, the chiral transformations $\delta_{5}$ are identical for all baryon fields within the same chiral representation, so we may define $g_{A}^{0}$ by

$$
\delta_{5} B=i \gamma_{5} \frac{\lambda^{0}{ }_{11} b_{0}}{2} g_{A}^{0} B=\frac{i \gamma_{5} b_{0}}{\sqrt{6}} g_{A}^{0} B
$$

where $B$ represents the baryon field, such as $\Lambda$ and $N_{1}^{N}-N_{2}^{N}$ etc.

2. For $g_{A}^{3}$, because $\lambda_{11}^{3}=-\lambda_{22}^{3}$, the chiral transformation $\delta_{5}^{b 3}$ is proportional to the isospin value of $\mathbf{I}_{\mathbf{z}}$, which is related to $\lambda^{3} / 2$. We factor it out from the definition of $g_{A}^{3}$ :

$$
\delta_{5}^{b 3} B=i \gamma_{5} b_{3} g_{A}^{3} \mathbf{I}_{\mathbf{z}} B+\cdots
$$

where $\operatorname{dots} \cdots$ on the right hand side contain off-diagonal terms.

3. For $g_{A}^{8}$, because $\lambda_{11}^{8}=\lambda_{22}^{8}$, the chiral transformations $\delta_{5}^{b 8}$ is the same for the baryon fields belonging to one isospin multiplet. We define it to be

$$
\delta_{5}^{b 8} B=i \gamma_{5} \frac{\lambda_{11}^{8} b_{8}}{2} g_{A}^{8} B+\cdots=\frac{i \gamma_{5} b_{8}}{2 \sqrt{3}} g_{A}^{8} B+\cdots
$$

The resulting axial coupling constants $g_{A}^{0}, g_{A}^{3}$ and $g_{A}^{8}$ are shown in Table III where $\Lambda$ is the (only) singlet field $\Lambda$; then $N_{-}, \Sigma_{-}, \Xi_{-}$and $\Lambda_{-}$are the octet fields of the type $N_{1}^{N}-N_{2}^{N}$; the $N_{+}, \Sigma_{+}, \Xi_{+}$and $\Lambda_{+}$are the octet fields of the type $N_{1}^{N}+N_{2}^{N}$; the $N_{\mu}, \Sigma_{\mu}, \Xi_{\mu}$ and $\Lambda_{\mu}$ are the octet fields $N_{\mu}^{N}$; the $\Delta_{\mu}, \Sigma_{\mu}^{*}, \Xi_{\mu}^{*}$ and $\Omega_{\mu}$ are the decuplet fields $\Delta_{\mu}^{P} ; \Delta_{\mu \nu}, \Sigma_{\mu \nu}^{*}, \Xi_{\mu \nu}^{*}$ and $\Omega_{\mu \nu}$ are the decuplet fields $\Delta_{\mu \nu}^{P}$.

From the values in Table III one can compute the $F$ and $D$ couplings easily. The resulting $F / D$ ratio,

$$
\alpha=\frac{g_{A}^{D}}{g_{A}^{F}+g_{A}^{D}}
$$

is also tabulated in the last column of Table III. Empirically, $\alpha \sim 0.6$, which is fairly close to the $S U(6)$ quark model value. In the present formalism we see that only the $(\mathbf{3}, \mathbf{6}) \oplus(\mathbf{6}, \mathbf{3})$ chiral multiplet/representation reproduces this 
TABLE III: Axial coupling constants $g_{A}^{0}, g_{A}^{3}$ and $g_{A}^{8}$. In the last column $\alpha=g_{A}^{D} /\left(g_{A}^{F}+g_{A}^{D}\right)$.

\begin{tabular}{|c|c|c|c|c|c|c|}
\hline$S U(3)_{L} \otimes S U(3)_{R}$ & $S U(3)_{F}$ & & $g_{A}^{0}$ & $g_{A}^{3}$ & $g_{A}^{8}$ & $\alpha$ \\
\hline \multirow{5}{*}{$(\overline{\mathbf{3}}, \mathbf{3}) \oplus(\mathbf{3}, \overline{\mathbf{3}})$} & 1 & $\bar{\Lambda}$ & \begin{tabular}{|l}
-1 \\
\end{tabular} & - & 0 & - \\
\hline & \multirow{4}{*}{8} & $N_{-}$ & -1 & 1 & -1 & \multirow{4}{*}{1} \\
\hline & & $\Sigma_{-}$ & \begin{tabular}{|l}
-1 \\
\end{tabular} & 0 & 2 & \\
\hline & & $\Xi_{-}$ & \begin{tabular}{|l|}
-1 \\
\end{tabular} & -1 & -1 & \\
\hline & & $\Lambda_{-}$ & -1 & - & -2 & \\
\hline \multirow{4}{*}{$(\mathbf{8}, \mathbf{1}) \oplus(\mathbf{1}, \mathbf{8})$} & \multirow{4}{*}{8} & $N_{+}$ & 3 & 1 & 3 & \multirow{4}{*}{0} \\
\hline & & $\Sigma_{+}$ & 3 & 1 & 0 & \\
\hline & & $\Xi_{+}$ & 3 & 1 & -3 & \\
\hline & & $\Lambda_{+}$ & 3 & - & 0 & \\
\hline \multirow{8}{*}{$(\mathbf{3}, \mathbf{6}) \oplus(\mathbf{6}, \mathbf{3})$} & \multirow{4}{*}{8} & $N_{\mu}$ & 1 & $5 / 3$ & 1 & \multirow{4}{*}{$3 / 5$} \\
\hline & & $\Sigma_{\mu}$ & 1 & $2 / 3$ & 2 & \\
\hline & & $\Xi_{\mu}$ & \begin{tabular}{|l|}
1 \\
\end{tabular} & $-1 / 3$ & -3 & \\
\hline & & $\Lambda_{\mu}$ & 1 & - & -2 & \\
\hline & \multirow{4}{*}{10} & $\Delta_{\mu}$ & 1 & $1 / 3$ & 1 & \\
\hline & & $\Sigma_{\mu}^{*}$ & \begin{tabular}{|l|}
1 \\
\end{tabular} & $1 / 3$ & 0 & \\
\hline & & $\Xi_{\mu}^{*}$ & 1 & $1 / 3$ & -1 & - \\
\hline & & $\Omega_{\mu}$ & 1 & - & -2 & \\
\hline \multirow{4}{*}{$(10,1) \oplus(1,10)$} & \multirow{4}{*}{10} & $\Delta_{\mu \nu}$ & 3 & 1 & 3 & \multirow{4}{*}{-} \\
\hline & & $\Sigma_{\mu \nu}^{*}$ & 3 & 1 & 0 & \\
\hline & & $\Xi_{\mu \nu}^{*}$ & \begin{tabular}{|l}
3 \\
\end{tabular} & 1 & -3 & \\
\hline & & $\Omega_{\mu \nu}$ & 3 & - & -6 & \\
\hline
\end{tabular}

value. Previous works have shown that this value is physically related to the coupling of the nucleon to the $\Delta(1232)$, as demonstrated in the Adler-Weisberger sum rule [28, 29]. This was also shown algebraically by Weinberg [1]. In both cases, saturation of the pion (axial-vector) induced transition from the nucleon to the $\Delta(1232)$ is essential [30]. In the present study, this is realized by the chiral representation which includes both the nucleon (isospin $1 / 2$ ) and delta (isospin 3/2) states.

It is also interesting that Table III shows that $g_{A}^{3}(N)=5 / 3, g_{A}^{0}(N)=1$ for $(\mathbf{3}, \mathbf{6}) \oplus(\mathbf{6}, \mathbf{3})$, while $g_{A}^{3}(N)=$ $1, g_{A}^{0}(N)=-1$ for $(\overline{\mathbf{3}}, \mathbf{3}) \oplus(\mathbf{3}, \overline{\mathbf{3}}) \cdot g_{A}^{0}$ corresponds to the so-called nucleon spin value, as measured in polarized deep inelastic scattering. A suitable superposition of the two chiral representations may improve the nucleon axial coupling in either the isovector and/or isosinglet sectors. The importance of such mixing for the isovector axial coupling constant has been emphasized by Weinberg since the late 1960-s, Ref. [1]. Here we have found the same result for the isovector, as well as extended it to the isosinglet part in a purely algebraic manner.

\section{SUMMARY}

In this paper we have performed a complete classification of flavor vector and chiral symmetries, and established several types of independent relativistic $S U(3)$ baryon interpolating fields. The three-quark fields may belong to one of several different Lorentz group representations which fact imposes certain constraints on possible chiral symmetry representations. This is due to the Pauli principle and has been explicitly verified by the method of Fierz transformations. As the present results reflect essentially the Pauli principle, they can be conveniently summarized as shown in Table IV by using the permutation symmetry group properties/representations. This table "explains" also the previous results for the case of isospin $S U(2)_{L} \times S U(2)_{R}$ [12]. From this table we have explicated the effective role of the Pauli principle in separate sectors of the left- and right-handed fermions.

In the real world, with spontaneous breaking of chiral symmetry, physical states of pure chiral (axial) symmetry representation do not occur, but in general they can mix in a state having a definite flavor symmetry. The present results show that the three-quark structures accommodate only a few (sometimes just one) chiral representations, for instance, for the total spin 1/2 field of Dirac spinor, the allowed chiral representations are two having the structure of Young tableaux $([21],-)$ and $([1],[11])$, where - indicates singlet. The $([21],-)$ representation corresponds respectively to $\left(\frac{1}{2}, 0\right)$ and $(8,1)$ of $S U(2)$ and $S U(3)$, whereas the $([1],[11])$ corresponds to $\left(\frac{1}{2}, 0\right)$ and $(3, \overline{3})$ of $S U(2)$ and $S U(3)$, respectively. Note that the chiral representations have the same structure as those of the Lorentz group. In this way, the Lorentz (spin) and flavor structures are combined into a structure of total permutation symmetry. As shown in the computation of $g_{A}$, in general, various couplings depend on the chiral representations with possible mixing. 
TABLE IV: Structure of allowed three-quark baryon fields.

\begin{tabular}{c|c|c|c|c|c}
\hline \hline Lorentz & J=Spin & $\begin{array}{c}\text { Young table } \\
\text { for Chiral rep. }\end{array}$ & Chiral $S U(2)$ & Chiral $S U(3)$ & Flavor $S U(3)$ \\
\hline$\left(\frac{1}{2}, 0\right) \oplus\left(0, \frac{1}{2}\right)$ & $1 / 2$ & $\begin{array}{c}([21],-) \oplus(-,[21]) \\
([1],[11]) \oplus([11],[1])\end{array}$ & $\left(\frac{1}{2}, 0\right) \oplus\left(0, \frac{1}{2}\right)$ & $\begin{array}{c}(8,1) \oplus(1,8) \\
(3, \overline{3}) \oplus(\overline{3}, 3)\end{array}$ & $\begin{array}{c}8 \\
1,8\end{array}$ \\
\hline$\left(1, \frac{1}{2}\right) \oplus\left(\frac{1}{2}, 1\right)$ & $3 / 2$ & $([2],[1]) \oplus([1],[2])$ & $\left(1, \frac{1}{2}\right) \oplus\left(\frac{1}{2}, 1\right)$ & $(6,3) \oplus(3,6)$ & 8,10 \\
\hline$\left(\frac{3}{2}, 0\right) \oplus\left(0, \frac{3}{2}\right)$ & $3 / 2$ & $([3],-) \oplus(-,[3])$ & $\left(\frac{3}{2}, 0\right) \oplus\left(0, \frac{3}{2}\right)$ & $(10,1) \oplus(1,10)$ & 10 \\
\hline
\end{tabular}

Such comparison may be useful for further understanding of the internal structure of hadrons in relation to chiral symmetry.

\section{Acknowledgments}

H.X.C. is grateful to Monkasho for their support of his stay at the Research Center for Nuclear Physics where this work was done. V.D and K.N thank Prof H. Toki for his hospitality during their stay at RCNP. A.H. is supported in part by the Grant for Scientific Research ((C) No.19540297) from the Ministry of Education, Culture, Science and Technology, Japan. K.N is supported by the National Science Council (NSC) of Republic of China under grant No. NSC96-2119-M-002-001. S.L.Z. was supported by the National Natural Science Foundation of China under Grants 10625521 and 10721063 and Ministry of Education of China.

\section{APPENDIX A: RARITA-SCHWINGER FIELDS}

In this appendix, we study the properties of Rarita-Schwinger fields, in the form of

$$
B_{\mu}(x) \sim \epsilon_{a b c}\left(q_{A}^{a T}(x) C \Gamma_{1} q_{B}^{b}(x)\right) \Gamma_{2} q_{C}^{c}(x),
$$

where there are eight possible pairs of $\Gamma_{1}$ and $\Gamma_{2}$,

$$
\begin{aligned}
\left(\Gamma_{1}, \Gamma_{2}\right)= & \left(\mathbf{1}, \gamma_{\mu}\right),\left(\gamma_{5}, \gamma_{\mu} \gamma_{5}\right),\left(\gamma_{\mu} \gamma_{5}, \gamma_{5}\right),\left(\gamma^{\nu} \gamma_{5}, \sigma_{\mu \nu} \gamma_{5}\right), \\
& \left(\gamma_{\mu}, \mathbf{1}\right),\left(\gamma^{\nu}, \sigma_{\mu \nu}\right),\left(\sigma_{\mu \nu}, \gamma^{\nu}\right),\left(\sigma_{\mu \nu} \gamma_{5}, \gamma^{\nu} \gamma_{5}\right) .
\end{aligned}
$$

The fields formed by these $\left(\Gamma_{1}, \Gamma_{2}\right)$ pairs are labeled by the subscript $i=(1, \cdots, 8)$ with the ordering of Eq. (A2). The discussion is separated into singlet, decuplet and octet cases.

\section{a. Flavor singlet baryon}

For flavor singlet fields, there are four apparently non-zero fields

$$
\begin{aligned}
& \Lambda_{1 \mu}=\epsilon_{a b c} \epsilon^{A B C}\left(q_{A}^{a T} C q_{B}^{b}\right) \gamma_{\mu} q_{C}^{c}, \\
& \Lambda_{2 \mu}=\epsilon_{a b c} \epsilon^{A B C}\left(q_{A}^{a T} C \gamma_{5} q_{B}^{b}\right) \gamma_{\mu} \gamma_{5} q_{C}^{c}, \\
& \Lambda_{3 \mu}=\epsilon_{a b c} \epsilon^{A B C}\left(q_{A}^{a T} C \gamma_{\mu} \gamma_{5} q_{B}^{b}\right) \gamma_{5} q_{C}^{c}, \\
& \Lambda_{4 \mu}=\epsilon_{a b c} \epsilon^{A B C}\left(q_{A}^{a T} C \gamma^{\nu} \gamma_{5} q_{B}^{b}\right) \sigma_{\mu \nu} \gamma_{5} q_{C}^{c} .
\end{aligned}
$$

As before, the Fierz transformed fields (primed fields) are just the corresponding unprimed ones, $\Lambda_{i \mu}^{\prime}=\Lambda_{i \mu}$. On the other hand, by applying the Fierz rearrangement (see Appendix. C), we obtain four equations

$$
\begin{aligned}
& \Lambda_{1 \mu}=-\frac{1}{4} \Lambda_{1 \mu}^{\prime}-\frac{1}{4} \Lambda_{2 \mu}^{\prime}+\frac{1}{4} \Lambda_{3 \mu}^{\prime}-\frac{i}{4} \Lambda_{4 \mu}^{\prime}, \\
& \Lambda_{2 \mu}=-\frac{1}{4} \Lambda_{1 \mu}^{\prime}-\frac{1}{4} \Lambda_{2 \mu}^{\prime}-\frac{1}{4} \Lambda_{3 \mu}^{\prime}+\frac{i}{4} \Lambda_{4 \mu}^{\prime}, \\
& \Lambda_{3 \mu}=\frac{1}{4} \Lambda_{1 \mu}^{\prime}-\frac{1}{4} \Lambda_{2 \mu}^{\prime}-\frac{1}{4} \Lambda_{3 \mu}^{\prime}-\frac{i}{4} \Lambda_{4 \mu}^{\prime}, \\
& \Lambda_{4 \mu}=\frac{3 i}{4} \Lambda_{1 \mu}^{\prime}-\frac{3 i}{4} \Lambda_{2 \mu}^{\prime}+\frac{3 i}{4} \Lambda_{3 \mu}^{\prime}+\frac{1}{4} \Lambda_{4 \mu}^{\prime} .
\end{aligned}
$$

Thus we find the following solution

$$
\Lambda_{1 \mu}=-\Lambda_{2 \mu}=\Lambda_{3 \mu}=-\frac{i}{3} \Lambda_{4 \mu}=\gamma_{\mu} \gamma_{5} \Lambda_{1}, \quad \Lambda_{6 \mu}=\Lambda_{7 \mu}=\Lambda_{8 \mu}=0
$$

We see that there is only one non-vanishing independent field. However, it has a structure of $\gamma_{\mu} \Lambda_{i}$. Therefore, they are all Dirac fields, and there is no flavor singlet fields of the Rarita-Schwinger type. 


\section{b. Flavor decuplet baryon}

For flavour decuplet fields, we have four potentially non-zero interpolators

$$
\begin{aligned}
\Delta_{5 \mu}^{P} & =\epsilon_{a b c} S_{P}^{A B C}\left(q_{A}^{a T} C \gamma_{\mu} q_{B}^{b}\right) q_{C}^{c}, \\
\Delta_{6 \mu}^{P} & =\epsilon_{a b c} S_{P}^{A B C}\left(q_{A}^{a T} C \gamma^{\nu} q_{B}^{b}\right) \sigma_{\mu \nu} q_{C}^{c}, \\
\Delta_{7 \mu}^{P} & =\epsilon_{a b c} S_{P}^{A B C}\left(q_{A}^{a T} C \sigma_{\mu \nu} q_{B}^{b}\right) \gamma^{\nu} q_{C}^{c}, \\
\Delta_{8 \mu}^{P} & =\epsilon_{a b c} S_{P}^{A B C}\left(q_{A}^{a T} C \sigma_{\mu \nu} \gamma_{5} q_{B}^{b}\right) \gamma^{\nu} \gamma_{5} q_{C}^{c} .
\end{aligned}
$$

As before, the Fierz transformed fields can be related to the corresponding unprimed ones, $\Delta_{i \mu}^{P \prime}=-\Delta_{i \mu}^{P}$. On the other hand, by applying the Fierz rearrangement to relate $\Delta_{i \mu}^{N}$ and $\Delta_{i \mu}^{N \prime}$, we obtain the solution

$$
\Delta_{5 \mu}^{P}=i \Delta_{6 \mu}^{P}=-i \Delta_{7 \mu}^{P}=i \Delta_{8 \mu}^{P} .
$$

There are no Dirac decuplet fields. Therefore, we obtain one extra non-vanishing field.

\section{c. Flavor octet baryon}

To study the octet baryon fields, we start with eight baryon fields:

$$
\begin{aligned}
& N_{1 \mu}^{N}=\epsilon_{a b c} \epsilon^{A B D} \lambda_{D C}^{N}\left(q_{A}^{a T} C q_{B}^{b}\right) \gamma_{\mu} q_{C}^{c}, \\
& N_{2 \mu}^{N}=\epsilon_{a b c} \epsilon^{A B D} \lambda_{D C}^{N}\left(q_{A}^{a T} C \gamma_{5} q_{B}^{b}\right) \gamma_{\mu} \gamma_{5} q_{C}^{c}, \\
& N_{3 \mu}^{N}=\epsilon_{a b c} \epsilon^{A B D} \lambda_{D C}^{N}\left(q_{A}^{a T} C \gamma_{\mu} \gamma_{5} q_{B}^{b}\right) \gamma_{5} q_{C}^{c}, \\
& N_{4 \mu}^{N}=\epsilon_{a b c} \epsilon^{A B D} \lambda_{D C}^{N}\left(q_{A}^{a T} C \gamma^{\nu} \gamma_{5} q_{B}^{b}\right) \sigma_{\mu \nu} \gamma_{5} q_{C}^{c}, \\
& N_{5 \mu}^{N}=\epsilon_{a b c} \epsilon^{A B D} \lambda_{D C}^{N}\left(q_{A}^{a T} C \gamma_{\mu} q_{B}^{b}\right) q_{C}^{c}=0, \\
& N_{6 \mu}^{N}=\epsilon_{a b c} \epsilon^{A B D} \lambda_{D C}^{N}\left(q_{A}^{a T} C \gamma^{\nu} q_{B}^{b}\right) \sigma_{\mu \nu} q_{C}^{c}=0, \\
& N_{7 \mu}^{N}=\epsilon_{a b c} \epsilon^{A B D} \lambda_{D C}^{N}\left(q_{A}^{a T} C \sigma_{\mu \nu} q_{B}^{b}\right) \gamma^{\nu} q_{C}^{c}=0, \\
& N_{8 \mu}^{N}=\epsilon_{a b c} \epsilon^{A B D} \lambda_{D C}^{N}\left(q_{A}^{a T} C \sigma_{\mu \nu} \gamma_{5} q_{B}^{b}\right) \gamma^{\nu} \gamma_{5} q_{C}^{c}=0 .
\end{aligned}
$$

There are four zero fields, but the corresponding Fierz transformed ones are non-zero. By using the Jacobi identity in Eq. (17), we obtain

$$
N_{1 \mu}^{N \prime}=-\frac{1}{2} N_{1 \mu}^{N}, N_{2 \mu}^{N \prime}=-\frac{1}{2} N_{2 \mu}^{N}, N_{3 \mu}^{N \prime}=-\frac{1}{2} N_{3 \mu}^{N}, N_{4 \mu}^{N \prime}=-\frac{1}{2} N_{4 \mu}^{N}
$$

Similarly, performing the Fierz transformation to relate $N_{i \mu}^{N}$ and $N_{i \mu}^{N \prime}$, we obtain the solution

$$
\begin{aligned}
& N_{4 \mu}^{N}=-i N_{1 \mu}^{N}+i N_{2 \mu}^{N}-i N_{3 \mu}^{N}, \\
& N_{5 \mu}^{N \prime}=-\frac{1}{2} N_{1 \mu}^{N}+\frac{1}{2} N_{2 \mu}^{N}-\frac{1}{2} N_{3 \mu}^{N}, \\
& N_{6 \mu}^{N \prime}=-i N_{1 \mu}^{N}+i N_{2 \mu}^{N}+\frac{i}{2} N_{3 \mu}^{N}, \\
& N_{7 \mu}^{N \prime}=i N_{1 \mu}^{N}+\frac{i}{2} N_{2 \mu}^{N}+i N_{3 \mu}^{N}, \\
& N_{8 \mu}^{N \prime}=\frac{i}{2} N_{1 \mu}^{N}+i N_{2 \mu}^{N}-i N_{3 \mu}^{N} .
\end{aligned}
$$

Thus we have shown that there are three different kinds of octets. However, $N_{1 \mu}^{N}$ and $N_{2 \mu}^{N}$ are nothing but $\gamma_{\mu} \gamma_{5} N_{1}^{N}$ and $\gamma_{\mu} \gamma_{5} N_{2}^{N}$ (see Eqs. (16)). Therefore, we only obtain one extra octet baryon field. It is formed by using the projection operator:

$$
P_{\mu \nu}^{3 / 2}=\left(g_{\mu \nu}-\frac{1}{4} \gamma_{\mu} \gamma_{\nu}\right),
$$

as

$$
\begin{aligned}
N_{\mu}^{N}=P_{\mu \nu}^{3 / 2} N_{3 \nu}^{N} & =\left(g_{\mu \nu}-\frac{1}{4} \gamma_{\mu} \gamma_{\nu}\right) \epsilon_{a b c} \epsilon^{A B D} \lambda_{D C}^{N}\left(q_{A}^{a T} C \gamma_{\mu} \gamma_{5} q_{B}^{b}\right) \gamma_{5} q_{C}^{c} \\
& =N_{3 \mu}^{N}+\frac{1}{4} \gamma_{\mu} \gamma_{5}\left(N_{1}^{N}-N_{2}^{N}\right)
\end{aligned}
$$




\section{APPENDIX B: TENSOR FIELDS}

In this appendix, we study the antisymmetric tensor baryons fields $J_{\mu \nu}$ with $J_{\mu \nu}=-J_{\nu \mu}$. For the tensor fields, we can form nine three-quark fields where the possible pairs of $\Gamma_{1}$ and $\Gamma_{2}$ are

$$
\begin{aligned}
\left(\Gamma_{1}, \Gamma_{2}\right)= & \left(\gamma_{\mu}, \gamma_{\nu} \gamma_{5}\right)-(\mu \leftrightarrow \nu),\left(\gamma_{\mu} \gamma_{5}, \gamma_{\nu}\right)-(\mu \leftrightarrow \nu), \\
& \epsilon_{\mu \nu \rho \sigma}\left(\gamma^{\rho}, \gamma^{\sigma}\right), \epsilon_{\mu \nu \rho \sigma}\left(\gamma^{\rho} \gamma_{5}, \gamma^{\sigma} \gamma_{5}\right),\left(\mathbf{1}, \sigma_{\mu \nu} \gamma_{5}\right),\left(\gamma_{5}, \sigma_{\mu \nu}\right), \\
& \left(\sigma_{\mu \nu}, \gamma_{5}\right),\left(\sigma_{\mu \nu} \gamma_{5}, \mathbf{1}\right), \epsilon_{\mu \nu \rho \sigma}\left(\sigma_{\rho l}, \sigma_{\sigma l}\right) .
\end{aligned}
$$

The fields formed by these $\left(\Gamma_{1}, \Gamma_{2}\right)$ pairs are labeled by the subscript $i=(1, \cdots, 9)$ with the ordering of Eq. (B1). The discussion is separated into singlet, decuplet and octet cases.

\section{d. Flavor singlet baryon}

The flavour singlet baryon fields have four potentially non-zero interpolators among nine fields:

$$
\begin{aligned}
& \Lambda_{2 \mu \nu}=\epsilon_{a b c} \epsilon^{A B C}\left(q_{A}^{a T} C \gamma_{\mu} \gamma_{5} q_{B}^{b}\right) \gamma_{\nu} q_{C}^{c}-(\mu \leftrightarrow \nu), \\
& \Lambda_{4 \mu \nu}=\epsilon_{a b c} \epsilon^{A B C} \epsilon_{\mu \nu \rho \sigma}\left(q_{A}^{a T} C \gamma_{\rho} \gamma_{5} q_{B}^{b}\right) \gamma_{\sigma} \gamma_{5} q_{C}^{c}, \\
& \Lambda_{5 \mu \nu}=\epsilon_{a b c} \epsilon^{A B C}\left(q_{A}^{a T} C q_{B}^{b}\right) \sigma_{\mu \nu} \gamma_{5} q_{C}^{c}, \\
& \Lambda_{6 \mu \nu}=\epsilon_{a b c} \epsilon^{A B C}\left(q_{A}^{a T} C \gamma_{5} q_{B}^{b}\right) \sigma_{\mu \nu} q_{C}^{c} .
\end{aligned}
$$

As before, the Fierz transformed fields are just the corresponding unprimed ones, $\Lambda_{i \mu \nu}^{\prime}=\Lambda_{i \mu \nu}$. On the other hand, by applying the Fierz rearrangement to relate $\Lambda_{i \mu \nu}$ and $\Lambda_{1 \mu \nu}^{\prime}$, we obtain the solution:

$$
i \Lambda_{2 \mu \nu}=\Lambda_{4 \mu \nu}=2 \Lambda_{5 \mu \nu}=-2 \Lambda_{6 \mu \nu} .
$$

Therefore, there is only one independent field. However, it has a structure of $\sigma_{\mu \nu} \Lambda_{i}$. Therefore, there are no extra fields.

\section{e. Flavor decuplet baryon}

The flavour decuplet baryon fields have five potentially non-zero interpolators:

$$
\begin{aligned}
\Delta_{1 \mu \nu}^{P} & =\epsilon_{a b c} S^{A B C}\left(q_{A}^{a T} C \gamma_{\mu} q_{B}^{b}\right) \gamma_{\nu} \gamma_{5} q_{C}^{c}-(\mu \leftrightarrow \nu), \\
\Delta_{3 \mu \nu}^{P} & =\epsilon_{a b c} S^{A B C} \epsilon_{\mu \nu \rho \sigma}\left(q_{A}^{a T} C \gamma_{\rho} q_{B}^{b}\right) \gamma_{\sigma} q_{C}^{c} \\
\Delta_{7 \mu \nu}^{P} & =\epsilon_{a b c} S^{A B C}\left(q_{A}^{a T} C \sigma_{\mu \nu} q_{B}^{b}\right) \gamma_{5} q_{C}^{c} \\
\Delta_{8 \mu \nu}^{P} & =\epsilon_{a b c} S^{A B C}\left(q_{A}^{a T} C \sigma_{\mu \nu} \gamma_{5} q_{B}^{b}\right) q_{C}^{c} \\
\Delta_{9 \mu \nu}^{P} & =\epsilon_{a b c} S^{A B C} \epsilon_{\mu \nu \rho \sigma}\left(q_{A}^{a T} C \sigma_{\rho l} q_{B}^{b}\right) \sigma_{\sigma l} q_{C}^{c} .
\end{aligned}
$$

As before, the Fierz transformed fields can be related to the corresponding unprimed ones, $\Delta_{i \mu \mu}^{P \prime}=-\Delta_{i \mu \mu}^{P}$. On the other hand, by applying the Fierz rearrangement to relate $\Delta_{i \mu \nu}^{P}$ and $\Delta_{i \mu \nu}^{P \prime}$, we obtain two independent fields: $\Delta_{1 \mu \nu}^{P}$ and $\Delta_{7 \mu \nu}^{P}$ :

$$
\Delta_{3 \mu \nu}^{P}=-i \Delta_{1 \mu \nu}^{P}, \Delta_{8 \mu \nu}^{P}=i \Delta_{1 \mu \nu}^{P}+\Delta_{7 \mu \nu}^{P}, \Delta_{9 \mu \nu}^{P}=-i \Delta_{1 \mu \nu}^{P}-2 \Delta_{7 \mu \nu}^{P}
$$

The first one $\Delta_{1 \mu \nu}^{P}$ can be related to the Rarita-Schwinger baryon fields, but the second one $\Delta_{7 \mu \nu}^{P}$ can not. Therefore, we obtain one extra decuplet fields. It is formed by using the projection operator:

$$
\Gamma^{\mu \nu \alpha \beta}=\left(g^{\mu \alpha} g^{\nu \beta}-\frac{1}{2} g^{\nu \beta} \gamma^{\mu} \gamma^{\alpha}+\frac{1}{2} g^{\mu \beta} \gamma^{\nu} \gamma^{\alpha}+\frac{1}{6} \sigma^{\mu \nu} \sigma^{\alpha \beta}\right),
$$

as

$$
\begin{aligned}
\Delta_{\mu \nu}^{P}=\Gamma^{\mu \nu \alpha \beta} \Delta_{7 \mu \nu}^{P} & =\Gamma^{\mu \nu \alpha \beta} \epsilon_{a b c} S^{A B C}\left(q_{A}^{a T} C \sigma_{\mu \nu} q_{B}^{b}\right) \gamma_{5} q_{C}^{c} \\
& =\Delta_{7 \mu \nu}^{P}-\frac{i}{2} \gamma_{\mu} \gamma_{5} \Delta_{5 \nu}^{P}+\frac{i}{2} \gamma_{\nu} \gamma_{5} \Delta_{5 \mu}^{P}
\end{aligned}
$$




\section{f. Flavor octet baryon}

To study the octet baryon fields, we start with nine octet baryon fields

$$
\begin{aligned}
& N_{1 \mu \nu}^{N}=\epsilon_{a b c} \epsilon^{A B D} \lambda_{D C}^{N}\left(q_{A}^{a T} C \gamma_{\mu} q_{B}^{b}\right) \gamma_{\nu} \gamma_{5} q_{C}^{c}-(\mu \leftrightarrow \nu)=0, \\
& N_{2 \mu \nu}^{N}=\epsilon_{a b c} \epsilon^{A B D} \lambda_{D C}^{N}\left(q_{A}^{a T} C \gamma_{\mu} \gamma_{5} q_{B}^{b}\right) \gamma_{\nu} q_{C}^{c}-(\mu \leftrightarrow \nu), \\
& N_{3 \mu \nu}^{N}=\epsilon_{a b c} \epsilon^{A B D} \lambda_{D C}^{N} \epsilon_{\mu \nu \rho \sigma}\left(q_{A}^{a T} C \gamma_{\rho} q_{B}^{b}\right) \gamma_{\sigma} q_{C}^{c}=0, \\
& N_{4 \mu \nu}^{N}=\epsilon_{a b c} \epsilon^{A B D} \lambda_{D C}^{N} \epsilon_{\mu \nu \rho \sigma}\left(q_{A}^{a T} C \gamma_{\rho} \gamma_{5} q_{B}^{b}\right) \gamma_{\sigma} \gamma_{5} q_{C}^{c}, \\
& N_{5 \mu \nu}^{N}=\epsilon_{a b c} \epsilon^{A B D} \lambda_{D C}^{N}\left(q_{A}^{a T} C q_{B}^{b}\right) \sigma_{\mu \nu} \gamma_{5} q_{C}^{c}, \\
& N_{6 \mu \nu}^{N}=\epsilon_{a b c} \epsilon^{A B D} \lambda_{D C}^{N}\left(q_{A}^{a T} C \gamma_{5} q_{B}^{b}\right) \sigma_{\mu \nu} q_{C}^{c}, \\
& N_{7 \mu \nu}^{N}=\epsilon_{a b c} \epsilon^{A B D} \lambda_{D C}^{N}\left(q_{A}^{a T} C \sigma_{\mu \nu} q_{B}^{b}\right) \gamma_{5} q_{C}^{c}=0, \\
& N_{8 \mu \nu}^{N}=\epsilon_{a b c} \epsilon^{A B D} \lambda_{D C}^{N}\left(q_{A}^{a T} C \sigma_{\mu \nu} \gamma_{5} q_{B}^{b}\right) q_{C}^{c}=0, \\
& N_{9 \mu \nu}^{N}=\epsilon_{a b c} \epsilon^{A B D} \lambda_{D C}^{N} \epsilon_{\mu \nu \rho \sigma}\left(q_{A}^{a T} C \sigma_{\rho l} q_{B}^{b}\right) \sigma_{\sigma l} q_{C}^{c}=0 .
\end{aligned}
$$

There are five zero fields, but the Fierz transformed ones are non-zero. By using the Jacobi identity in Eq. (17), we obtain

$$
N_{2 \mu \nu}^{N \prime}=-\frac{1}{2} N_{2 \mu \nu}^{N}, N_{4 \mu \nu}^{N \prime}=-\frac{1}{2} N_{4 \mu \nu}^{N}, N_{5 \mu \nu}^{N \prime}=-\frac{1}{2} N_{5 \mu \nu}^{N}, N_{6 \mu \nu}^{N \prime}=-\frac{1}{2} N_{6 \mu \nu}^{N}
$$

Similarly, performing the Fierz transformation to relate $N_{i \mu \nu}^{N}$ and $N_{i \mu \nu}^{N \prime}$, we find that there are three independent fields $N_{2 \mu \nu}^{N}, N_{5 \mu \nu}^{N}$ and $N_{6 \mu \nu}^{N}$. Here are the relations:

$$
\begin{aligned}
& N_{4 \mu \nu}^{N}=-i N_{2 \mu \nu}^{N}-N_{5 \mu \nu}^{N}+N_{6 \mu \nu}^{N}, \\
& N_{1 \mu \nu}^{N \prime}=-\frac{1}{2} N_{2 \mu \nu}^{N}+i N_{5 \mu \nu}^{N}-i N_{6 \mu \nu}^{N}, \\
& N_{3 \mu \nu}^{N \prime}=\frac{i}{2} N_{2 \mu \nu}^{N}-\frac{1}{2} N_{5 \mu \nu}^{N}+\frac{1}{2} N_{6 \mu \nu}^{N}, \\
& N_{7 \mu \nu}^{N \prime}=-\frac{i}{2} N_{2 \mu \nu}^{N}-\frac{1}{2} N_{5 \mu \nu}^{N}, \\
& N_{8 \mu \nu}^{N \prime}=\frac{i}{2} N_{2 \mu \nu}^{N}-\frac{1}{2} N_{6 \mu \nu}^{N}, \\
& N_{9 \mu \nu}^{N \prime}=-N_{5 \mu \nu}^{N}-N_{6 \mu \nu}^{N} .
\end{aligned}
$$

All these three fields can be related to the Dirac spinor and Rarita-Schwinger fields. Therefore, there are no extra octet fields.

\section{APPENDIX C: FIERZ TRANSFORMATION}

In this appendix, we list the Fierz transformations used in our calculation, which are proved by using Mathematica [27]. Here we would like to show only the change in the structure of Lorentz indices of direct products of two Dirac matrices under the Fierz rearrangement. Therefore, in the following equations, we do not include the minus sign which arises from the exchange of quark fields. The formulae go for the three cases corresponding to the Dirac, Rarita-Schwinger and tensor fields when applied to three-quark fields.

1. Products of two Dirac matrices without Lorentz indices:

$$
\left(\begin{array}{l}
\mathbf{1} \otimes \gamma_{5} \\
\gamma_{\mu} \otimes \gamma^{\mu} \gamma_{5} \\
\sigma_{\mu \nu} \otimes \sigma^{\mu \nu} \gamma_{5} \\
\gamma_{\mu} \gamma_{5} \otimes \gamma^{\mu} \\
\gamma_{5} \otimes \mathbf{1}
\end{array}\right)_{a b, c d}=\left(\begin{array}{lllll}
\frac{1}{4} & -\frac{1}{4} & \frac{1}{8} & \frac{1}{4} & \frac{1}{4} \\
-1 & -\frac{1}{2} & 0 & -\frac{1}{2} & 1 \\
3 & 0 & -\frac{1}{2} & 0 & 3 \\
1 & -\frac{1}{2} & 0 & -\frac{1}{2} & -1 \\
\frac{1}{4} & \frac{1}{4} & \frac{1}{8} & -\frac{1}{4} & \frac{1}{4}
\end{array}\right)\left(\begin{array}{l}
\mathbf{1} \otimes \gamma_{5} \\
\gamma_{\mu} \otimes \gamma^{\mu} \gamma_{5} \\
\sigma_{\mu \nu} \otimes \sigma^{\mu \nu} \gamma_{5} \\
\gamma_{\mu} \gamma_{5} \otimes \gamma^{\mu} \\
\gamma_{5} \otimes \mathbf{1}
\end{array}\right)_{a d, b c}
$$

2. Products of two Dirac matrices with one Lorentz index:

$$
\left(\begin{array}{l}
\mathbf{1} \otimes \gamma^{\mu} \\
\gamma^{\mu} \otimes \mathbf{1} \\
\gamma_{5} \otimes \gamma_{\mu} \gamma_{5} \\
\gamma_{\mu} \gamma_{5} \otimes \gamma_{5} \\
\gamma^{\nu} \otimes \sigma_{\mu \nu} \\
\sigma_{\mu \nu} \otimes \gamma^{\nu} \\
\gamma^{\nu} \gamma_{5} \otimes \sigma_{\mu \nu} \gamma_{5} \\
\sigma_{\mu \nu} \gamma_{5} \otimes \gamma^{\nu} \gamma_{5}
\end{array}\right)_{a b, c d}=\left(\begin{array}{llllllll}
\frac{1}{4} & \frac{1}{4} & \frac{1}{4} & -\frac{1}{4} & -\frac{i}{4} & \frac{i}{4} & \frac{i}{4} & \frac{i}{4} \\
\frac{1}{4} & \frac{1}{4} & -\frac{1}{4} & \frac{1}{4} & \frac{i}{4} & -\frac{i}{4} & \frac{i}{4} & \frac{i}{4} \\
\frac{1}{4} & -\frac{1}{4} & \frac{1}{4} & \frac{1}{4} & \frac{i}{4} & \frac{i}{4} & -\frac{i}{4} & \frac{i}{4} \\
-\frac{1}{4} & \frac{1}{4} & \frac{1}{4} & \frac{1}{4} & \frac{i}{4} & \frac{i}{4} & \frac{i}{4} & -\frac{i}{4} \\
\frac{3 i}{4} & -\frac{3 i}{4} & -\frac{3 i}{4} & -\frac{3 i}{4} & -\frac{1}{4} & -\frac{1}{4} & -\frac{1}{4} & \frac{1}{4} \\
-\frac{3 i}{4} & \frac{3 i}{4} & -\frac{3 i}{4} & -\frac{3 i}{4} & -\frac{1}{4} & -\frac{1}{4} & \frac{1}{4} & -\frac{1}{4} \\
-\frac{3 i}{4} & -\frac{3 i}{4} & \frac{3 i}{4} & -\frac{3 i}{4} & -\frac{1}{4} & \frac{1}{4} & -\frac{1}{4} & -\frac{1}{4} \\
-\frac{3 i}{4} & -\frac{3 i}{4} & -\frac{3 i}{4} & \frac{3 i}{4} & \frac{1}{4} & -\frac{1}{4} & -\frac{1}{4} & -\frac{1}{4}
\end{array}\right)\left(\begin{array}{l}
\mathbf{1} \otimes \gamma^{\mu} \\
\gamma^{\mu} \otimes \mathbf{1} \\
\gamma_{5} \otimes \gamma_{\mu} \gamma_{5} \\
\gamma_{\mu} \gamma_{5} \otimes \gamma_{5} \\
\gamma^{\nu} \otimes \sigma_{\mu \nu} \\
\sigma_{\mu \nu} \otimes \gamma^{\nu} \\
\gamma^{\nu} \gamma_{5} \otimes \sigma_{\mu \nu} \gamma_{5} \\
\sigma_{\mu \nu} \gamma_{5} \otimes \gamma^{\nu} \gamma_{5}
\end{array}\right)_{a d, b c}
$$


3. Products of two Dirac matrices with two anti-symmetric Lorentz indices:

$$
\left(\begin{array}{l}
\mathbf{1} \otimes \sigma_{\mu \nu} \gamma_{5} \\
\gamma_{5} \otimes \sigma_{\mu \nu} \\
\sigma_{\mu \nu} \otimes \gamma_{5} \\
\sigma_{\mu \nu} \gamma_{5} \otimes \mathbf{1} \\
\epsilon_{\mu \nu \rho \sigma} \sigma_{\rho l} \otimes \sigma_{\sigma l} \\
\gamma_{\mu} \otimes \gamma_{\nu} \gamma_{5}-(\mu \leftrightarrow \nu) \\
\gamma_{\mu} \gamma_{5} \otimes \gamma_{\nu}-(\mu \leftrightarrow \nu) \\
\epsilon_{\mu \nu \rho \sigma} \gamma_{\rho} \otimes \gamma_{\sigma} \\
\epsilon_{\mu \nu \rho \sigma} \gamma_{\rho} \gamma_{5} \otimes \gamma_{\sigma} \gamma_{5}
\end{array}\right)_{a b, c d}=\left(\begin{array}{lllllllll}
\frac{1}{4} & \frac{1}{4} & \frac{1}{4} & \frac{1}{4} & \frac{1}{4} & \frac{i}{4} & -\frac{i}{4} & \frac{1}{4} & -\frac{1}{4} \\
\frac{1}{4} & \frac{1}{4} & \frac{1}{4} & \frac{1}{4} & \frac{1}{4} & -\frac{i}{4} & \frac{i}{4} & -\frac{1}{4} & \frac{1}{4} \\
\frac{1}{4} & \frac{1}{4} & \frac{1}{4} & \frac{1}{4} & -\frac{1}{4} & -\frac{i}{4} & \frac{i}{4} & \frac{1}{4} & -\frac{1}{4} \\
\frac{1}{4} & \frac{1}{4} & \frac{1}{4} & \frac{1}{4} & -\frac{1}{4} & \frac{i}{4} & -\frac{i}{4} & -\frac{1}{4} & \frac{1}{4} \\
1 & 1 & -1 & -1 & 0 & 0 & 0 & 0 & 0 \\
-\frac{i}{2} & \frac{i}{2} & \frac{i}{2} & -\frac{i}{2} & 0 & 0 & 0 & \frac{i}{2} & \frac{i}{2} \\
\frac{i}{2} & -\frac{i}{2} & -\frac{i}{2} & \frac{i}{2} & 0 & 0 & 0 & \frac{i}{2} & \frac{i}{2} \\
\frac{1}{2} & -\frac{1}{2} & \frac{1}{2} & -\frac{1}{2} & 0 & -\frac{i}{2} & -\frac{i}{2} & 0 & 0 \\
-\frac{1}{2} & \frac{1}{2} & -\frac{1}{2} & \frac{1}{2} & 0 & -\frac{i}{2} & -\frac{i}{2} & 0 & 0
\end{array}\right)\left(\begin{array}{l}
\mathbf{1} \otimes \sigma_{\mu \nu} \gamma_{5} \\
\gamma_{5} \otimes \sigma_{\mu \nu} \\
\sigma_{\mu \nu} \otimes \gamma_{5} \\
\sigma_{\mu \nu} \gamma_{5} \otimes \mathbf{1} \\
\epsilon_{\mu \nu \rho \sigma} \sigma_{\rho l} \otimes \sigma_{\sigma l} \\
\gamma_{\mu} \otimes \gamma_{\nu} \gamma_{5}-(\mu \leftrightarrow \nu) \\
\gamma_{\mu} \gamma_{5} \otimes \gamma_{\nu}-(\mu \leftrightarrow \nu) \\
\epsilon_{\mu \nu \rho \sigma} \gamma_{\rho} \otimes \gamma_{\sigma} \\
\epsilon_{\mu \nu \rho \sigma} \gamma_{\rho} \gamma_{5} \otimes \gamma_{\sigma} \gamma_{5}
\end{array}\right)(\mathrm{C} 3)
$$

\section{APPENDIX D: CHIRAL PROPERTIES OF RARITA-SCHWINGER FIELDS}

In this appendix, we study the chiral properties of Rarita-Schwinger fields. As previously described in Section IV. we only need to study the properties of $(L L) L,(L L) R,(L R) L$ and $(R L) L$.

\section{Chiral properties of $(L L) L$}

The chiral representations of $(L L) L$ are $(\mathbf{1}, \mathbf{1}) \oplus(\mathbf{8}, \mathbf{1}) \oplus(\mathbf{8}, \mathbf{1}) \oplus(\mathbf{1 0}, \mathbf{1})$. We will study them separately in the following.

(1) In principle, there are eight possibilities of making the Rarita-Schwinger fields as shown in Eq. (A2). However, the chiral representation $(\mathbf{1}, \mathbf{1})$ has just two non-zero fields:

$$
\begin{aligned}
& \Lambda_{L 1 \mu}=\epsilon_{a b c} \epsilon^{A B C}\left(L_{A}^{a T} C L_{B}^{b}\right) \gamma_{\mu} L_{C}^{c}, \\
& \Lambda_{L 2 \mu}=\epsilon_{a b c} \epsilon^{A B C}\left(L_{A}^{a T} C \gamma_{5} L_{B}^{b}\right) \gamma_{\mu} \gamma_{5} L_{C}^{c} .
\end{aligned}
$$

Similarly performing the Fierz transformation to relate $\Lambda_{L i \mu}$ and $\Lambda_{L i \mu}^{\prime}$, we obtain the solution that all such kind of fields vanish.

(2) The chiral representation $(\mathbf{1 0}, \mathbf{1})$ has two non-zero fields:

$$
\begin{aligned}
\Delta_{L 7 \mu}^{P} & =\epsilon_{a b c} S_{P}^{A B C}\left(L_{A}^{a T} C \sigma_{\mu \nu} L_{B}^{b}\right) \gamma^{\nu} L_{C}^{c}, \\
\Delta_{L 8 \mu}^{P} & =\epsilon_{a b c} S_{P}^{A B C}\left(L_{A}^{a T} C \sigma_{\mu \nu} \gamma_{5} L_{B}^{b}\right) \gamma^{\nu} \gamma_{5} L_{C}^{c} .
\end{aligned}
$$

Similarly performing the Fierz transformation to relate $\Delta_{L i \mu}^{P}$ and $\Delta_{L i \mu}^{P \prime}$, we obtain the solution that all such kind of fields vanish.

(3) The chiral representation $(\mathbf{8}, \mathbf{1})$ has two non-zero fields:

$$
\begin{aligned}
& N_{L 1 \mu}^{N}=\epsilon_{a b c} \epsilon^{A B D} \lambda_{D C}^{N}\left(L_{A}^{a T} C L_{B}^{b}\right) \gamma_{\mu} L_{C}^{c} \\
& N_{L 2 \mu}^{N}=\epsilon_{a b c} \epsilon^{A B D} \lambda_{D C}^{N}\left(L_{A}^{a T} C \gamma_{5} L_{B}^{b}\right) \gamma_{\mu} \gamma_{5} L_{C}^{c}
\end{aligned}
$$

Similarly performing the Fierz transformation to relate $N_{L i \mu}^{N}$ and $N_{L i \mu}^{N \prime}$, we obtain the solution

$$
N_{L 2 \mu}^{N}=N_{L 1 \mu}^{N}
$$

Others are just zero. There is only one non-vanishing octet baryon field.

\section{Chiral properties of $(L L) R,(L R) L$ and $(R L) L$}

The chiral representations of $(L L) R,(L R) L$ and $(R L) L$ are $(\overline{\mathbf{3}}, \mathbf{3}) \oplus(\mathbf{6}, \mathbf{3})$. We will study them separately in the following.

(1) The chiral representation $(\overline{\mathbf{3}}, \mathbf{3}) \rightarrow \mathbf{1}_{\mathbf{f}}$ has two non-zero components:

$$
\begin{aligned}
& \Lambda_{M 1 \mu}=\epsilon_{a b c} \epsilon^{A B C}\left(L_{A}^{a T} C L_{B}^{b}\right) \gamma_{\mu} R_{C}^{c} \\
& \Lambda_{M 2 \mu}=\epsilon_{a b c} \epsilon^{A B C}\left(L_{A}^{a T} C \gamma_{5} L_{B}^{b}\right) \gamma_{\mu} \gamma_{5} R_{C}^{c} .
\end{aligned}
$$


Similarly performing the Fierz transformation to relate $\Lambda_{M i \mu}$ and $\Lambda_{M i \mu}^{\prime}$, we obtain the solution

$$
\Lambda_{M 1 \mu}=-\Lambda_{M 2 \mu} .
$$

Others are just zero. There is only one non-vanishing field. Others $(L R) L$ and $(R L) L$ can be related to this one.

(2) The chiral representation $(\mathbf{6}, \mathbf{3}) \rightarrow \mathbf{1 0}_{\mathbf{f}}$ has two non-zero components:

$$
\begin{aligned}
& \Delta_{M 7 \mu}^{P}=\epsilon_{a b c} S^{A B C}\left(L_{A}^{a T} C \sigma_{\mu \nu} L_{B}^{b}\right) \gamma^{\nu} R_{C}^{c}, \\
& \Delta_{M 8 \mu}^{P}=\epsilon_{a b c} S^{A B C}\left(L_{A}^{a T} C \sigma_{\mu \nu} \gamma_{5} L_{B}^{b}\right) \gamma^{\nu} \gamma_{5} R_{C}^{c} .
\end{aligned}
$$

Others are just zero. Similarly performing the Fierz transformation to relate $\Delta_{M i \mu}^{P}$ and $\Delta_{M i \mu}^{P \prime}$, we obtain the solution

$$
\Delta_{M 7 \mu}^{P}=-\Delta_{M 8 \mu}^{P} .
$$

There is only one non-vanishing field. Others $(L R) L$ and $(R L) L$ can be related to this one.

(3) The chiral representations $(\overline{\mathbf{3}}, \mathbf{3}) \rightarrow \mathbf{8}_{\mathbf{f}}$ has only two non-zero interpolators:

$$
\begin{aligned}
& N_{M 1 \mu}^{N}=\epsilon_{a b c} \epsilon^{A B D} \lambda_{D C}^{N}\left(L_{A}^{a T} C L_{B}^{b}\right) \gamma_{\mu} R_{C}^{c}, \\
& N_{M 2 \mu}^{N}=\epsilon_{a b c} \epsilon^{A B D} \lambda_{D C}^{N}\left(L_{A}^{a T} C \gamma_{5} L_{B}^{b}\right) \gamma_{\mu} \gamma_{5} R_{C}^{c} .
\end{aligned}
$$

Similarly performing the Fierz transformation to relate $N_{M i \mu}^{N}$ and $N_{M i \mu}^{N \prime}$, we obtain the solution

$$
N_{M 1 \mu}^{N}=-N_{M 2 \mu}^{N} .
$$

In order to study the chiral representations $(\mathbf{6}, \mathbf{3}) \rightarrow \mathbf{8}_{\mathbf{f}}$, we need to consider the second way (see the discussion in Section IIB3 which leads to four non-zero fields:

$$
\begin{aligned}
& \widetilde{N}_{M 1 \mu}^{N}=\epsilon_{a b c} \epsilon^{A C D} \lambda_{D B}^{N}\left(L_{A}^{a T} C L_{B}^{b}\right) \gamma_{\mu} R_{C}^{c}, \\
& \widetilde{N}_{M 2 \mu}^{N}=\epsilon_{a b c} \epsilon^{A C D} \lambda_{D B}^{N}\left(L_{A}^{a T} C \gamma_{5} L_{B}^{b}\right) \gamma_{\mu} \gamma_{5} R_{C}^{c}, \\
& \widetilde{N}_{M 7 \mu}^{N}=\epsilon_{a b c} \epsilon^{A C D} \lambda_{D B}^{N}\left(L_{A}^{a T} C \sigma_{\mu \nu} L_{B}^{b}\right) \gamma^{\nu} R_{C}^{c}, \\
& \widetilde{N}_{M 8 \mu}^{N}=\epsilon_{a b c} \epsilon^{A C D} \lambda_{D B}^{N}\left(L_{A}^{a T} C \sigma_{\mu \nu} \gamma_{5} L_{B}^{b}\right) \gamma^{\nu} \gamma_{5} R_{C}^{c} .
\end{aligned}
$$

By using the Jacobi identity in Eq. (17), we obtain:

$$
\widetilde{N}_{M 1 \mu}^{N}=\frac{1}{2} N_{M 1 \mu}^{N}, \widetilde{N}_{M 2 \mu}^{N}=\frac{1}{2} N_{M 2 \mu}^{N} .
$$

Similarly performing the Fierz transformation to relate $\widetilde{N}_{M i \mu}^{N}$ and $\widetilde{N}_{M i \mu}^{N \prime}$, we obtain the solution

$$
\begin{aligned}
& \widetilde{N}_{M 2 \mu}^{N}=-\widetilde{N}_{M 1 \mu}^{N}=-\frac{1}{2} N_{M 1 \mu}^{N}, \\
& \widetilde{N}_{M 8 \mu}^{N}=-\widetilde{N}_{M 7 \mu}^{N} .
\end{aligned}
$$

All together there are two non-vanishing independent fields: $\widetilde{N}_{M 1 \mu}^{N}$ and $\widetilde{N}_{M 7 \mu}^{N}$. $\widetilde{N}_{M 1 \mu}^{N}$ is related to $N_{M 1 \mu}^{N}$, and so belongs to the chiral representation $((\overline{\mathbf{3}}, \mathbf{3}))$. However, the other $\widetilde{N}_{M 7 \mu}^{N}$ can not be related to $N_{M i \mu}^{N}$, and so contains some $(6,3)$ component. other Others $(L R) L$ and $(R L) L$ can be related to $(L L) R$. Chiral properties of the tensor fields can be also explored in completely the same procedure explained here. Therefore, we do not show this case any more.

[1] S. Weinberg, Phys. Rev. 177, 2604 (1969); Phys. Rev. Lett. 65, 1177 (1990).

[2] T. Hatsuda and T. Kunihiro, Phys. Rept. 247, 221 (1994).

[3] B. W. Lee, Chiral Dynamics, (Gordon and Breach, New York, 1972).

[4] T. D. Lee, Particle Physics and Introduction to Field Theory, (Harwood, Chur, Switzerland, 1981).

[5] D. Jido, M. Oka and A. Hosaka, Prog. Theor. Phys. 106, 873 (2001).

[6] D. Jido, M. Oka and A. Hosaka, Phys. Rev. Lett. 80, 448 (1998).

[7] D. Jido, T. Hatsuda and T. Kunihiro, Phys. Rev. Lett. 84, 3252 (2000). 
[8] S. R. Beane and M. J. Savage, Phys. Lett. B 556, 142 (2003).

[9] M. Benmerrouche, R. M. Davidson and N. C. Mukhopadhyay, Phys. Rev. C 39, 2339 (1989).

[10] H. Haberzettl, arXiv:nucl-th/9812043.

[11] C. DeTar and T. Kunihiro, Phys. Rev. D 39, 2805 (1989).

[12] K. Nagata, A. Hosaka and V. Dmitrasinovic, arXiv:0705.1896 [hep-ph].

[13] B. L. Ioffe, Nucl. Phys. B 188, 317 (1981) [Erratum-ibid. B 191, 591 (1981)]; B. L. Ioffe, Z. Phys. C 18, 67 (1983).

[14] Y. Chung, H. G. Dosch, M. Kremer and D. Schall, Nucl. Phys. B 197 (1982) 55.

[15] D. Espriu, P. Pascual and R. Tarrach, Nucl. Phys. B 214 (1983) 285.

[16] F. X. Lee and X. Y. Liu, Phys. Rev. D 66, 014014 (2002).

[17] D. B. Leinweber, W. Melnitchouk, D. G. Richards, A. G. Williams and J. M. Zanotti, Lect. Notes Phys. 663, 71 (2005).

[18] J. M. Zanotti, D. B. Leinweber, A. G. Williams, J. B. Zhang, W. Melnitchouk and S. Choe [CSSM Lattice collaboration], Phys. Rev. D 68, 054506 (2003).

[19] T. D. Cohen and X. D. Ji, Phys. Rev. D 55, 6870 (1997).

[20] H. X. Chen, A. Hosaka and S. L. Zhu, Phys. Rev. D 74, 054001 (2006).

[21] S. Okubo, Prog. Theor. Phys. 27, 949 (1962).

[22] S. Ogawa, S. Sawada, and M. Nakagawa, Composite Models of Elementary Particles, (in russian, Mir, Moscow, USSR, 1983).

[23] R. E. Marshak, N. Mukunda and S. Okubo, Phys. Rev. 137, B698 (1965).

[24] Y. Hara, Phys. Rev. 139, B134 (1965).

[25] J. Schechter and Y. Ueda, Phys. Rev. 144, 1338 (1966).

[26] G. A. Christos, Phys. Rev. D 35, 330 (1987).

[27] J. A. Maruhn, T. Buervenich and D. G. Madland, arXiv:nucl-th/0007010

[28] S.L. Adler, Phys. Rev. 140. B736 (1965).

[29] W.I. Weissberger, Phys. Rev. 143, 1302 (1966).

[30] J. F. Donoghue and D. Wyler, Phys. Rev. D 17, 280 (1978). 